\title{
Multi-season optical modulation phased with the orbit of the super-Earth 55 Cancri e
}

\author{
S. Sulis ${ }^{1}$, D. Dragomir ${ }^{2,3}$, M. Lendl ${ }^{1,4}$, V. Bourrier ${ }^{4}$, B. O. Demory ${ }^{5}$, L. Fossati ${ }^{1}$, P. E. Cubillos ${ }^{1}$, \\ D. B. Guenther ${ }^{6}$, S. R. Kane ${ }^{7}$, R. Kuschnig ${ }^{8}$, J. M. Matthews ${ }^{9}$, A. F. J. Moffat ${ }^{10}$, J. F. Rowe ${ }^{11}$, \\ D. Sasselov ${ }^{12}$, W. W. Weiss ${ }^{8}$, and J. N. Winn ${ }^{13}$ \\ ${ }^{1}$ Space Research Institute, Austrian Academy of Sciences, Schmiedlstraße 6, 8042 Graz, Austria \\ e-mail: sophia.sulis@oeaw.ac.at \\ 2 Department of Physics and Kavli Institute for Astrophysics and Space Research, Massachusetts Institute of Technology, Cambridge, \\ MA 02139, USA \\ ${ }^{3}$ Department of Physics and Astronomy, University of New Mexico, Albuquerque, NM, USA \\ ${ }^{4}$ Observatoire de l'Université de Genève, 51 chemin des Maillettes, 1290 Sauverny, Switzerland \\ ${ }^{5}$ University of Bern, Center for Space and Habitability, Sidlerstraße 5, 3012 Bern, Switzerland \\ ${ }^{6}$ Institute for Computational Astrophysics, Department of Astronomy and Physics, Saint Mary's University, Halifax, \\ NS B3H 3C3, Canada \\ ${ }^{7}$ Department of Earth and Planetary Sciences, University of California, Riverside, CA 92521, USA \\ ${ }^{8}$ Institut für Astronomie, Universität Wien Türkenschanzstrasse 17, 1180 Wien, Austria \\ ${ }^{9}$ Department of Physics and Astronomy, University of British Columbia, 6224 Agricultural Road, Vancouver, \\ BC V6T 1Z1, Canada \\ ${ }^{10}$ Observatoire Astronomique du Mont Mégantic, Departement de Physique, Université de Montréal C. P. 6128, \\ Succursale: Centre-Ville, Montréal, QC H3C 3J7, Canada \\ ${ }^{11}$ NAS Ames Research Park, Mail Stop 244-30, Moffett Field, CA 94035-1000, USA \\ ${ }^{12}$ Harvard-Smithsonian Center for Astrophysics, 60 Garden Street, Cambridge, MA 102138, USA \\ ${ }^{13}$ Department of Astrophysical Sciences, Princeton University, 4 Ivy Lane, Princeton, NJ 08544, USA
}

Received 11 June 2019 / Accepted 24 September 2019

\begin{abstract}
Context. $55 \mathrm{Cnc}$ e is a transiting super-Earth orbiting a solar-like star with an orbital period of $\sim 17.7 \mathrm{~h}$. In 2011 , using the Microvariability and Oscillations in Stars (MOST) space telescope, a quasi-sinusoidal modulation in flux was detected with the same period as the planetary orbit. The amplitude of this modulation was too large to be explained as the change in light reflected or emitted by the planet.

Aims. The MOST telescope continued to observe $55 \mathrm{Cnc}$ e for a few weeks per year over five years (from 2011 to 2015), covering 143 individual transits. This paper presents the analysis of the observed phase modulation throughout these observations and a search for the secondary eclipse of the planet.

Methods. The most important source of systematic noise in MOST data is due to stray-light reflected from the Earth, which is modulated with both the orbital period of the satellite $(101.4 \mathrm{~min})$ and the Earth's rotation period. We present a new technique to deal with this source of noise, which we combined with standard detrending procedures for MOST data. We then performed Markov chain Monte Carlo analyses of the detrended light curves, modeling the planetary transit and phase modulation.

Results. We find phase modulations similar to those seen in 2011 in most of the subsequent years; however, the amplitude and phase of maximum light are seen to vary, from year to year, from 113 to $28 \mathrm{ppm}$ and from 0.1 to $3.8 \mathrm{rad}$. The secondary eclipse is not detected, but we constrain the geometric albedo of the planet to less than $0.47(2 \sigma)$.

Conclusions. While we cannot identify a single origin of the observed optical modulation, we propose a few possible scenarios. Those include star-planet interaction, such as coronal rains and spots rotating with the motion of the planet along its orbit, or the presence of a transiting circumstellar torus of dust. However, a detailed interpretation of these observations is limited by their photometric precision. Additional observations at optical wavelengths could measure the variations at higher precision, contribute to uncovering the underlying physical processes, and measure or improve the upper limit on the albedo of the planet.
\end{abstract}

Key words. planetary systems - techniques: photometric - stars: individual: 55 Cancri

\section{Introduction}

Though super-Earths as a category of exoplanets have been discussed for nearly a decade, the nature and origins of these planets are diverse (Rogers et al. 2011; Hansen \& Murray 2012; Mordasini et al. 2012; Chiang \& Laughlin 2013; Batygin \& Laughlin 2015; Dorn et al. 2018). A wide range of compositions are possible for these planets, whose mass and size lie between those of the Earth and Neptune (Adams et al. 2008; Rogers \& Seager 2010). Super-Earths are among the most numerous planets within the sample of detected planets, even though there are no analogs to these planets in our solar system (Mayor et al. 2011; Petigura et al. 2013; Fressin et al. 2013). Recent studies found that, within this size regime, the planet size distribution is bimodal and has a gap between 1.5 and $2.0 R_{\oplus}$ (Fulton et al. 2017; Fulton \& Petigura 2018). The most widespread explanation 
for this "radius gap" is photoevaporation (Lecavelier des Etangs 2007; Davis \& Wheatley 2009; Ehrenreich \& Désert 2011). In this scenario, planets for which the $\mathrm{H} / \mathrm{He}$ atmosphere constitutes less than about $1 \%$ of the total mass at the time of the dispersal of the protoplanetary disk are fated to lose this atmosphere completely within about 100 Myr. In contrast, planets with initially more massive atmospheres are able to retain enough gas to cause the mean density to be substantially lower than that of a purely rocky planet. The former have completely lost their primary, hydrogen-dominated atmosphere, and therefore their (small) radius depends exclusively on the average density of the rocky core. For the latter, instead, because of the low planetary mass (i.e., low gravity) and low atmospheric mean molecular weight, the envelope extends far from the rocky surface leading to a larger planetary radius (Owen \& Wu 2017; Jin \& Mordasini 2018; Van Eylen et al. 2018). The planet 55 Cnc e itself is located at a very short orbital distance and exposed to more intense stellar radiation than the bulk of planets making up the populations discussed above. This planet is an example of only a handful objects with low masses and high irradiation often referred to as ultra-short period planets (USPs).

With an orbital period of just 0.736 days, the super-Earth $55 \mathrm{Cnc}$ e is an extremely hot, presumably tidally locked superEarth with a brightness temperature of $\sim 2700 \pm 270 \mathrm{~K}$ (Demory et al. 2016a). It orbits the third brightest star ( $V$ mag $=5.95$; TESS mag $=5.48$ ) known to host a transiting exoplanet in this size category (Winn et al. 2011; Demory et al. 2011a) after HD 39091 (Jones et al. 2002; Gandolfi et al. 2018) and HD 219134 (Motalebi et al. 2015; Vogt et al. 2015). Based on transit and radial velocity data, the radius of $55 \mathrm{Cnc}$ e is $1.88 \pm 0.03 R_{\oplus}$ and the mass is $8.0 \pm 0.3 M_{\oplus}$ (Bourrier et al. 2018a). The high bulk density is $6.7 \pm 0.4 \mathrm{~g} \mathrm{~cm}^{-3}$. Given these measurements, it is not yet possible to tell whether the planet has an Earth-like composition (an iron core surrounded by a silicate mantle) or a rocky core with an envelope of volatiles. Atmospheric escape models for such a small, highly irradiated planet predict that there should be no substantial H/He envelope (Gillon et al. 2012; Demory et al. 2016a; Kubyshkina et al. 2018) - and indeed no hydrogen exosphere has been detected (Ehrenreich et al. 2012). However, given its bulk density, the planet is most likely surrounded by a heavyweight atmosphere (Bourrier et al. 2018a).

This scenario is supported by Spitzer $4.5 \mu \mathrm{m}$ phase curve observations (Demory et al. 2016a). These observations show an eastward offset of the hot spot of the planet and a night temperature of $1380 \pm 400 \mathrm{~K}$; both of these characteristics require some heat circulation. If $55 \mathrm{Cnc}$ e were a "lava planet" with no atmosphere, it is unlikely that its heat redistribution efficiency would be sufficiently high to explain these two features (Kite et al. 2016; Angelo \& Hu 2017). It seems more likely that $55 \mathrm{Cnc}$ e has an optically thick atmosphere, as suggested by Demory et al. (2016a), Angelo \& Hu (2017), and later by Bourrier et al. (2018a) through refined values of the planet mass and radius. The presence of a high-metallicity atmosphere is also indicated by the tentative detection of $\mathrm{Ca}^{+}$and $\mathrm{Na}$ in the planet exosphere (Ridden-Harper et al. 2016). The present-day atmosphere may well be shrouding a molten surface, and might have originated from volcanic outgassing.

A quasi-sinusoidal modulation in the optical flux of the $55 \mathrm{Cnc}$ e system was detected using data from the Microvariability and Oscillations in Stars (MOST) space telescope (Winn et al. 2011, hereafter W11). The modulation had the same period as the planet and an amplitude initially measured at $168 \pm 70 \mathrm{ppm}$. While small, this amplitude is too large to be due to only scattered light from the planet, which cannot exceed $\sim 30 \mathrm{ppm}$ in the
MOST bandpass. For the more massive giant planet HD 20782b, similar MOST observations revealed the signature of reflected light from the planetary atmosphere as the planet passed through periastron (Kane et al. 2016). Therefore, W11 suggested instead that the modulation was some sort of instrumental artifact, or that it may represent a previously unknown type of star-planet interaction.

Since $55 \mathrm{Cnc}$ e is not sufficiently massive to give rise to observable signatures of tidal interaction in the MOST photometry, the flux modulation at the period of $55 \mathrm{Cnc}$ e may indicate the existence of magnetic interactions between the planet and its host star. A scenario involving interactions between the stellar corona activity and the planet has already been proposed by Bourrier et al. (2018b) to explain some modulation in flux observed in UV data as well. Located in a very close orbit, $55 \mathrm{Cnc}$ e is an ideal target for detecting interactions that consist of an active region on the stellar surface rotating with the planet orbital motion instead of the stellar rotation period (Shkolnik et al. 2003; Walker et al. 2008; Poppenhaeger \& Schmitt 2011; Lanza 2012; Strugarek et al. 2015, 2019; Shkolnik \& Llama 2018; Wright \& Miller 2015; Cauley et al. 2018).

Other analyses for $55 \mathrm{Cnc}$ e, based on IR observations of the secondary eclipse have indicated variability in its secondary eclipse depth (Demory et al. 2016b; Tamburo et al. 2018). The authors found a $4 \sigma$ difference in eclipse depth between two epochs of observations (acquired in 2012 and 2013, respectively). Demory et al. (2016b) suggested either volcanic activity or an inhomogeneous circumstellar torus of gas and dust as possible explanations. Alternatively, Tamburo et al. (2018) proposed that the planet may be intermittently covered by reflective grains originating from volcanic activity or cloud variability.

$55 \mathrm{Cnc}$ e has already benefited from numerous multiwavelength observations in the IR (Demory et al. 2011a, 2016b,a), UV (Bourrier et al. 2018b), and optical (W11; Gillon et al. 2012; Dragomir et al. 2014). In this paper we present an extensive dataset of optical photometry obtained with MOST, most of which has not been previously published. We use these data to provide new clues to the nature of this mysterious planet, by monitoring the time variable nature of this system, constraining the albedo of planet e and searching for transits of the other four known planets in the system. We describe the MOST observations and their reduction in Sects. 2 and 3. The data analysis, results on the refined transit parameters, and discussion regarding 55 Cnc e are found in Sects. 4 and 6, respectively. In Sect. 5 we present a search for transits of the other known planets in the system, and we conclude in Sect. 7.

\section{MOST observations of 55 Cnc}

The MOST telescope (Walker et al. 2003; Matthews 2004) is a now inactive microsatellite carrying a $15 \mathrm{~cm}$ optical telescope, which acquires light through a broadband filter spanning the visible wavelengths from 350 to $700 \mathrm{~nm}$. This instrument remains in a Sun-synchronous polar orbit with a period of $101.4 \mathrm{~min}$, which allowed it to monitor stars in a continuous viewing zone (CVZ) without interruption for up to eight weeks. The CVZ covered a declination range from $+36^{\circ}>\delta>-18^{\circ}$. Stars brighter than $V \sim 5-6$ were observed using a Fabry microlens to project onto the CCD an image of the telescope pupil illuminated by the target. Fainter stars were observed in direct imaging mode, in which the defocused images of the stars were projected onto the CCD (Rowe et al. 2006).

$55 \mathrm{Cnc}$ was in the CVZ of MOST, and was observed every year from 2011 to 2015 with timespans between about 15 and 
Table 1. Dates, durations, exposure times, and initial number of transits $\left(N_{\text {tr }}\right)$ corresponding to the MOST observations taken between 2011 and 2015 .

\begin{tabular}{|c|c|c|c|c|c|}
\hline \multirow{2}{*}{$\begin{array}{c}\text { Standard } \\
\text { year }\end{array}$} & \multicolumn{2}{|c|}{ Dates (BJD) } & \multirow{2}{*}{$\begin{array}{c}\text { Duration } \\
\text { (days) }\end{array}$} & \multirow{2}{*}{$\begin{array}{c}\text { Integration } \\
\text { Time (s) }\end{array}$} & \multirow{2}{*}{$N_{t r}$} \\
\hline & Start & End & & & \\
\hline 2011 & 5599.52 & 5614.50 & 14.98 & 40 & 18 \\
\hline 2012 & 5940.51 & 5982.67 & 42.16 & 40 & 52 \\
\hline 2013 & 6328.78 & 6348.70 & 19.93 & 40 & 27 \\
\hline 2014 & 6689.10 & 6713.52 & 24.43 & 60 & 25 \\
\hline 2015 & 7033.64 & 7074.50 & 40.86 & 120 & 53 \\
\hline
\end{tabular}

Notes. Dates are given in barycentric Julian days (BJD) to within a constant 2450000 .

42 days. The observations were acquired in direct imaging mode with an exposure time of $0.5 \mathrm{~s}$ per individual frame. The images were downloaded from the satellite in stacks of 40-240, resulting in total integration times ranging from 20 to $120 \mathrm{~s}$ (excluding overheads) per downloaded data point. Including overheads, the sampling rate ranged from 20.91 to $124.44 \mathrm{~s}$. Table 1 shows the dates, duration, and exposure times used for each of the five time series.

Raw light curves were extracted from the images using aperture photometry. We found that an aperture radius of five pixels almost always gave the lowest scatter in the residuals, so for consistency we used this size to extract all of the MOST photometry. We detail the reduction and analysis of the light curves in the next section.

\section{Photometric analysis}

There are several challenges in reducing MOST observations to obtain the final light curves (Rowe et al. 2006). In the steps detailed below, we independently reduce each of the five datasets taken between 2011 and 2015. We note that the first two datasets (2011-2012) have already been presented in W11 and Dragomir et al. (2014; hereafter D14). The detrending steps used in the present study follow established methods used to reduce MOST datasets (Rowe et al. 2006; W11; D14).

\subsection{Data pre-whitening}

The first step consists in removing the extreme outliers exceeding ten standard deviations $(\sigma)$ from the median flux. To obtain a homogeneous time series for each dataset, and also because we observed correlations between the significant outliers and different integration times, we removed data points with integration times differing from the values given in Table 1.

For each year, we fit the entire dataset (15-42 days) with a fifth degree polynomial function of both the sky background and pixel-to-pixel shifts. We then divided the time series by the best-fit polynomials to obtain a corrected, normalized sequence. Then, we again eliminated outliers lying above $5 \sigma$ from the median flux $(<0.5 \%$ of the time series).

After these steps, we removed some parts of the light curves that are affected by large observational gaps (mainly due to tracking lost). We find that doing so increases the signal-to-noise ratio $(\mathrm{S} / \mathrm{N})$ of the detected transits. Indeed, observations surrounding these data gaps show a particularly large number of outliers compared to the remaining values. For the 2011 dataset, we removed the first 0.6 and last 2.1 days (as done in W11), for 2012 we removed the first 0.5 and last 5.85 days, for 2013 only the last 2.67 days, for 2014 the first 8.31 and last 0.643 days, and for 2015 the first 10.71 days (see Appendix A).

In the resulting sequences, we observed a long-term variation that may be due to stellar activity; the stellar rotation period is 38.8 days (Bourrier et al. 2018a). This variation is shown and discussed in Appendix. A. This had to be corrected before we could make the final correction of patterns related to the satellite motion (see Sect. 3.2). We proceeded as follows: we first masked transits and secondary eclipse, and then binned the observations into intervals of twice the planet orbital period. Then, we fit a spline function to the binned data, and removed it from the initial unbinned sequence. We have investigated several alternatives to this technique (e.g., boxcar, Gaussian or Savitzky \& Golay 1964 filters) but, as long as we consider filter sizes larger than twice the planetary period, all of these approaches lead to similar results.

\subsection{Correction for stray-light flux variations}

The MOST satellite completed one polar orbit around Earth in $P_{\text {sat }}=101.4 \mathrm{~min}$. Its observations are affected by the scattered Earthshine, which generates flux variations with amplitudes that vary from orbit to orbit depending on the part of the Earth visible to the satellite. These variations are modulated with both the orbital period of the satellite and the Earth's rotation period. An example of a pattern observed at $P_{\text {sat }}$ is shown in the top left panel of Fig. 1, while the sinusoidal-like pattern observed at the Earth's rotation period is shown in the top middle panel. As discussed in W11, the stray-light timescales and amplitudes are too different from the orbital period of planet e to mimic the variations observed at the planetary period (see Sect. 4.2). However, the orbital period of the satellite is comparable to the planet transit duration $(\approx 95 \mathrm{~min})$ and the correction of the straylight patterns should be done carefully to avoid any influence on the inferred transit parameters.

\subsubsection{Classical method}

The shape of the variations induced by stray-light is variable from one satellite orbit to another. In panel a of Fig. 2, we show the one-day sequences of the 2012 dataset phase folded on the satellite orbital period. Each of these shorter sequences contains approximately $14 P_{\text {sat }}$. We observe a variability in the shape of the pattern and a time delay between the distinct features. These variations illustrate a need to treat MOST observations on a similar timescale. Traditional techniques (Rucinski et al. 2004; Rowe et al. 2008; Dragomir et al. 2013) consist in correcting these stray-light systematics on short sequences (e.g., of two-day length). Typically, the short time series are phase folded at the satellite orbital period and a moving average filter is iteratively removed for each of them. In this paper, we performed a similar technique to correct for the stray-light variations, which is detailed below:

1. select a one-day time-series (corresponding to $\sim 14 P_{\text {sat }}$ );

2. mask transits and eclipses;

3. phase fold on satellite orbital period;

4. use a Savitzky-Golay (SG) filter of window size $w_{1}$ (instead of a moving average) to model the detailed variability at the MOST period;

5. remove this variability from the entire one-day sequence (including transits and eclipses);

6. unfold the subseries;

7. repeat steps 1-6 for each one-day sequence. 

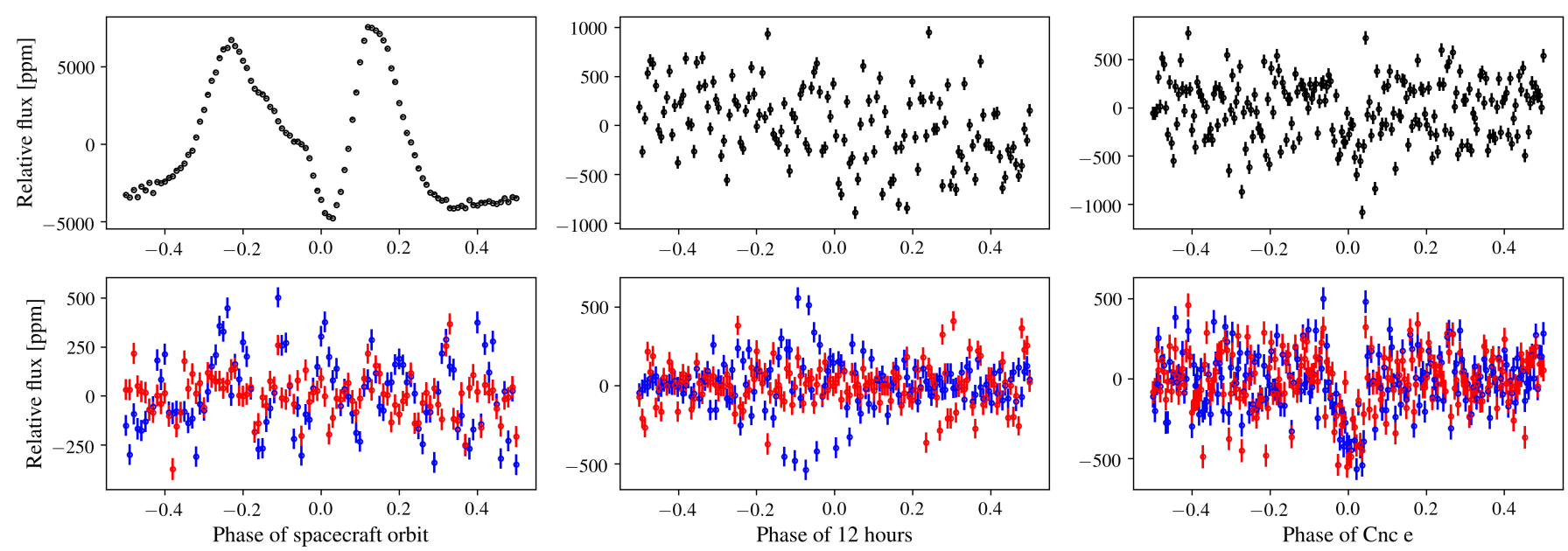

Fig. 1. MOST data obtained in 2015 after pre-whitening (top panels, see Sect. 3.1) and after the correction of the Earth stray-light variations (bottom panels, see Sect. 3.2). We show the relative flux phase folded at the satellite period (left, binned into $1 \mathrm{~min}$ ), a period of $12 \mathrm{~h}$ (middle, binned into $5 \mathrm{~min}$ ), and the planet period (right, binned into $5 \mathrm{~min}$ ). In the bottom panels, observations detrended by the classical procedure (see Sect. 3.2.1) are shown in blue and by the time-shift procedure (see Sect. 3.2.2) in red. In these plots, the uncertainties are based on the original unscaled photometric uncertainties.
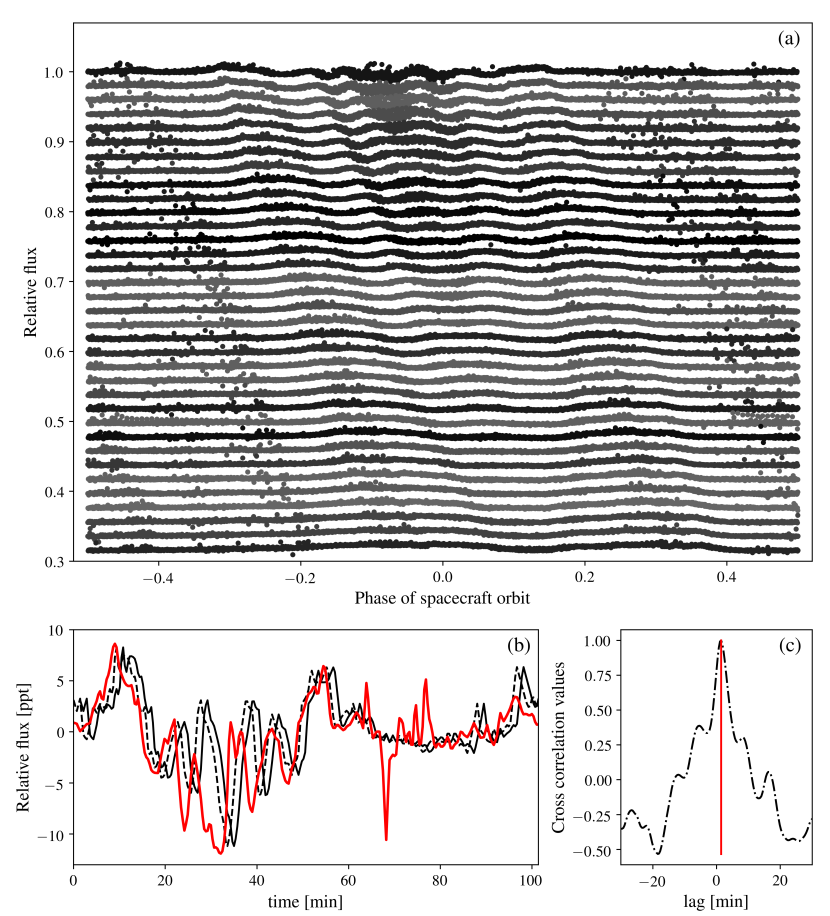

Fig. 2. Illustration of the Earth stray-light variation with respect to the orbital period of the satellite (2012 dataset). (a) Relative flux of the oneday sequences phase folded at the MOST satellite period and binned over 5-min intervals. The different series have been shifted in flux for visibility. The shape of the stray-light evolves from day-to-day and a time delay is observed. (b) Example of patterns with a duration of one satellite period belonging to the same one-day time series. The $y$-axis is in part-per-thousand. The solid lines show an unshifted (black) and the reference (red) pattern. The dashed line shows the black pattern corrected by the estimated time delay $(-86 \mathrm{~s})$, which was found using the cross-correlation function shown in $(c)$.

Then, as some correlated noise remains, we removed the final structures related to the orbital period of the satellite and the Earth's rotation period as follows:

8. mask transits and occultations from the entire light curve;

9. phase fold the series at the MOST orbital period;
Table 2. Parameters involved in the classical and time-shift detrending procedures.

\begin{tabular}{|c|c|c|c|c|c|c|}
\hline & Year & 2011 & 2012 & 2013 & 2014 & 2015 \\
\hline \multirow{5}{*}{ 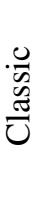 } & $w_{1}^{(a)}$ & 27 & 7 & 7 & 27 & 7 \\
\hline & $w_{2}^{(a)}$ & 331 & 681 & 837 & 341 & 801 \\
\hline & $w_{3}^{(a)}$ & 357 & 751 & 957 & 217 & 57 \\
\hline & $\mathrm{rms}$ & 1016 & 1031 & 722 & 915 & 1213 \\
\hline & $S / N$ & 13.9 & 26.7 & 20.9 & 15.9 & 10.2 \\
\hline \multirow{8}{*}{ 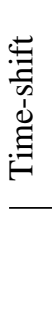 } & $w_{1}^{(a)}$ & 27 & 39 & 91 & 43 & 27 \\
\hline & $w_{2}^{(a)}$ & 261 & 861 & 131 & 321 & 711 \\
\hline & $w_{3}^{(a)}$ & 2111 & 121 & 2031 & 671 & 171 \\
\hline & $\mathrm{rms}$ & 995 & 1084 & 773 & 561 & 1162 \\
\hline & $S / N$ & 13.9 & 26.8 & 21.2 & 16.4 & 9.4 \\
\hline & $N_{\mathrm{pts}}$ & 21236 & 67445 & 32521 & 17811 & 18757 \\
\hline & $T_{\mathrm{obs}}$ & 12 & 35 & 17 & 14 & 29 \\
\hline & $N_{\mathrm{tr}}$ & 16 & 47 & 23 & 18 & 39 \\
\hline
\end{tabular}

Notes. ${ }^{(a)}$ SG window widths have to be uneven, the rms units are ppm, and $T_{\mathrm{obs}}$ is in days.

10. use a SG filter (of width $w_{2}$ ) to model the residual variability at the satellite period;

11. remove this variability from the entire light curve (including transits and eclipses);

12. repeat steps $8-11$ for the satellite period harmonic $P_{\text {sat }} / 2$ (using width $w_{2}$ ), the Earth's rotation period and its $12 \mathrm{~h}$ harmonic (using width $w_{3}$ );

13. unfold the series and remove the final $3 \sigma$ outliers from the median value.

We chose the different parameters involved in this procedure (length of the subseries, window sizes of the filters, and sigma clipping level) such that they maximize the final S/N of the known planetary transits (evaluated using Eq. (2) of Pont et al. 2006). Table 2 lists the best window widths $\left\{w_{1}, w_{2}, w_{3}\right\}$ found during this procedure, the final root-mean-square (rms) measured out-of-transit and the transit $\mathrm{S} / \mathrm{N}$ for the different 

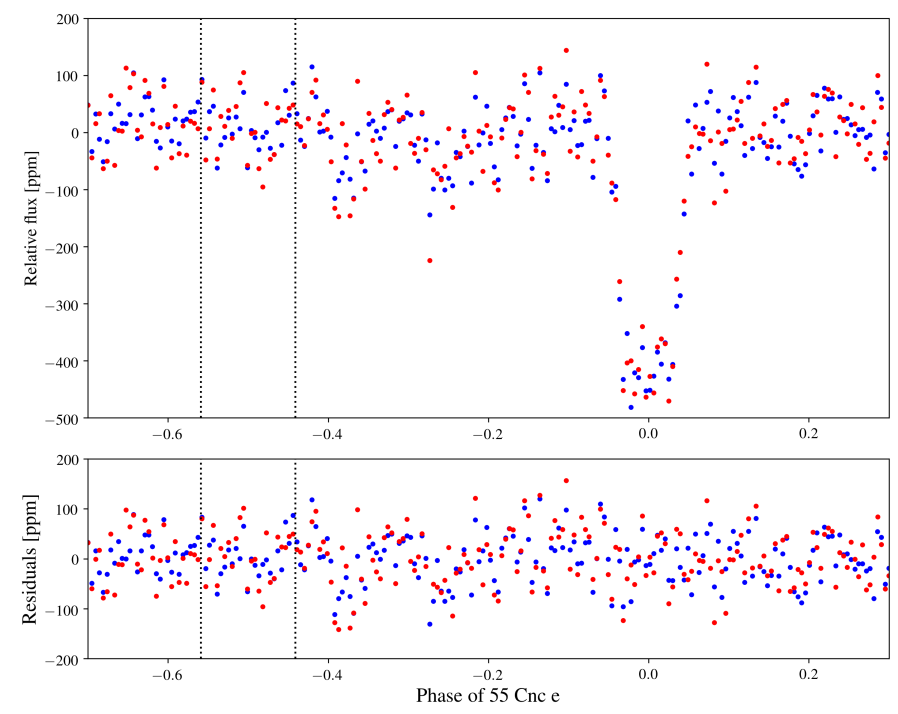

Fig. 3. Combine light curve phase folded at the planet orbital period and binned into 5-min intervals (top) and associated residuals (bottom). The observed phase modulation has been removed using results given in Sect. 4.2 to compare only the transit shape. Observations detrended by the classical procedure are shown in blue and by the time-shift procedure in red. At the timescale of the planet orbital period, the light curves are globally identical $(S / N \sim 40, \mathrm{rms} \sim 1000 \mathrm{ppm})$. The phase range of the secondary eclipse are indicated by the vertical dotted lines.

sequences (first horizontal box). The final length of the observations $\left(T_{\mathrm{obs}}\right)$, the number of data points $\left(N_{\mathrm{pts}}\right)$, and the number of transit events $\left(N_{\mathrm{tr}}\right)$ are also indicated at the end of the table. When the datasets are combined, we count 157770 data points and 143 transit events.

The bottom panels of Fig. 1 show this light curve detrending procedure (blue lines), illustrating how stray-light variations related to $P_{\text {sat }}$ (left) and the Earth rotation (middle) are removed. Figure 3 shows the combined light curve phase folded at the planetary orbital period and Fig. 4 shows each sequence between 2011 and 2015 (left column).

The detrended light curve corresponding to the 2011 dataset (top left panel of Fig. 4) is comparable with that reduced by W11 using a similar detrending technique based on moving average filters. However, we obtain a slightly higher scatter than W11 at the transit location owing to the transit masking steps added to the procedure described above (steps 3 and 8).

\subsubsection{Improvement of the traditional detrending method: time-shift procedure}

We propose an alternative method to improve the modeling of the stray-light pattern modulated at the satellite orbital period (step 4 of the classical procedure). As shown in panel a of Fig. 2, we observe a variability in both the shape of the pattern and a time delay between the distinct features.

However, smaller time delays are also present between individual orbits during the one-day sequences shown in Fig. 2. To take these into account, we developed a new technique that consists in cross-correlating each of the 14 individual $P_{\text {sat }}$ features with a reference sequence; because of small data gaps we used the sequence with the largest number of data points. During this step, both transits and eclipses are masked. After compensating the time delay for each of the sequences, we isolated the general pattern using a SG filter (width $w_{1}$ ) and removed it from the considered one-day time series (containing transits and eclipses).
Then, we applied steps 5-12 of the classical procedure described above. An example of the time delay between two consecutive patterns is shown by the black and red solid lines in panel $b$ of Fig. 2. A normalized cross-correlation function derived from two of these short series is shown in panel $\mathrm{c}$, where we find a time shift of $-86 \mathrm{~s}$. The dashed black curve in panel b shows the black feature shifted by this delay to match the reference feature.

The middle box in Table 2 lists the parameters involved in this new procedure as well as its performance in terms of rms and transit $\mathrm{S} / \mathrm{N}$. While the increase (resp. decrease) of the transit $\mathrm{S} / \mathrm{N}$ (resp. rms) is not drastic, the benefit of this procedure can be seen in the comparison of the final light curves. The 2015 light curve phase folded at the spacecraft orbital period resulting from this time-shift procedure is shown in red in the bottom right panel of Fig. 1. We see that, even if it does not increase the transit S/N (see last column of Table 2), the time-shift method significantly reduces the systematics induced by stray-light occurring at the timescale of the spacecraft orbit. The light curves phase folded at the planet orbital period are shown in the middle panel of Fig. 4. Comparing these light curves with those obtained from the classical procedure (Fig. 4, left column), we see a reduction of the remaining pattern modulated with the orbital period of the satellite $(\sim 1 / 10$ of the planet period). This effect is particularly remarkable for the 2011 dataset (top panels). However, as we see in Sect. 4, this correction does not significantly affect the features occurring at the timescale of the planet orbital period $\left(>10 P_{\text {sat }}\right)$. At this timescale, the light curves detrended by both procedures remain comparable with a similar transit $\mathrm{S} / \mathrm{N}$ when the light curves are combined ( $\mathrm{S} / \mathrm{N}$ of 40.2 , see Fig. 3). A better visualization of the detected modulation in flux is shown in Fig. 5 for the light curves detrended by the time-shift procedure with the transit model removed.

We finally note that the time-shift method could also be applied on the whole time series to correct the time delay between all orbits globally. However, as the shape of the stray-light pattern changes significantly over the course of several days, this would degrade the accuracy of the correction.

\section{Transit properties of $55 \mathrm{Cnc} e$}

To interpret our data, we performed a Markov chain Monte Carlo (MCMC) analysis using both the light curves detrended via the classical method, with and without the time-shift correction. A detailed description of the MCMC scheme can be found in Lendl et al. (2017). We used the Mandel \& Agol (2002) algorithm to model transits and occultations, and the differentialevolution MCMC engine described in Cubillos et al. (2017). As our datasets do not have a high enough $\mathrm{S} / \mathrm{N}$ to fit for stellar limb darkening, we used a quadratic limb-darkening law with fixed parameters that have been derived for the MOST bandpass: $u_{1}=0.648$ and $u_{2}=0.117$ (D14). To derive the transit, phase variation and secondary eclipse parameters, we carried out our analysis in three steps.

First, we fit for the transit period $(P)$, epoch of mid-transit $\left(T_{0}\right)$, planet-to-star radius ratio $\left(R_{\mathrm{p}} / R_{\mathrm{S}}\right)$, impact parameter $(b)$, and transit duration $\left(t_{\mathrm{d}}\right)$ to estimate precisely the planetary orbital period. Second, we fixed the orbital period and proceeded to a second fit of the light curve, combining the transit model with a function modeling the variations in flux observed at the planetary period (see Fig. 4). We added these variations to the transit model as sinusoidal functions of (fixed) planetary orbital frequency $f_{\mathrm{s}}=1 / P$ :

$F_{\text {mod }}(t)=\alpha_{\text {mod }} \sin \left(2 \pi f_{\mathrm{s}} t+\phi_{\text {mod }}\right)$, 

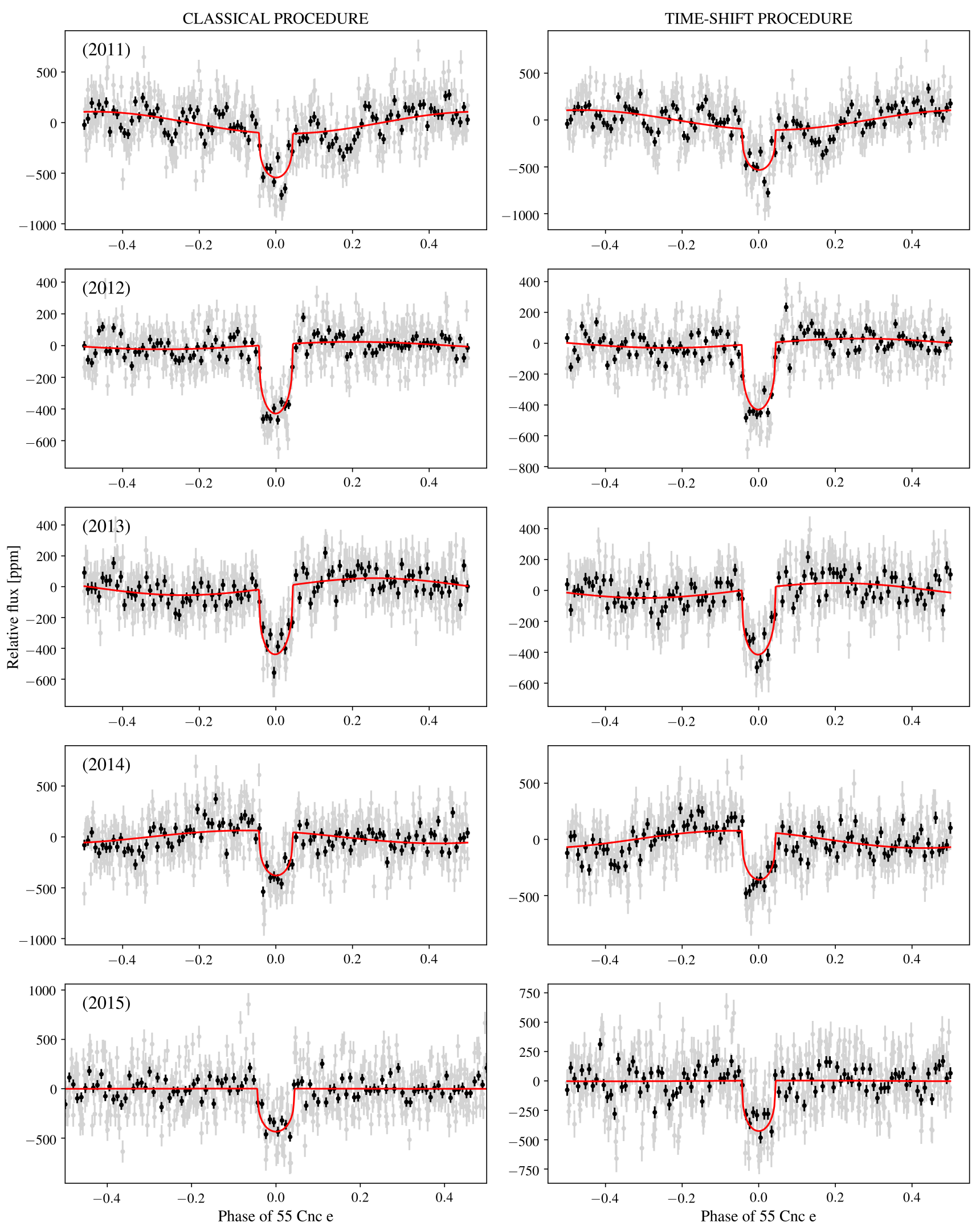

Fig. 4. Light curves phase folded at the planet orbital period and binned into 2 (gray) and 10-min (black) intervals. From top to bottom: data taken in 2011, 2012, 2013, 2014, and 2015. First column: final light curves obtained via the classical detrending procedure and the second column shows the light curves obtained via the time-shift detrending procedure. In these plots, the uncertainties are based on the original unscaled photometric uncertainties (in contrast to the scaled errors used in the MCMC analyses, see Sect. 4.4). The best-fitting models are shown in red. 

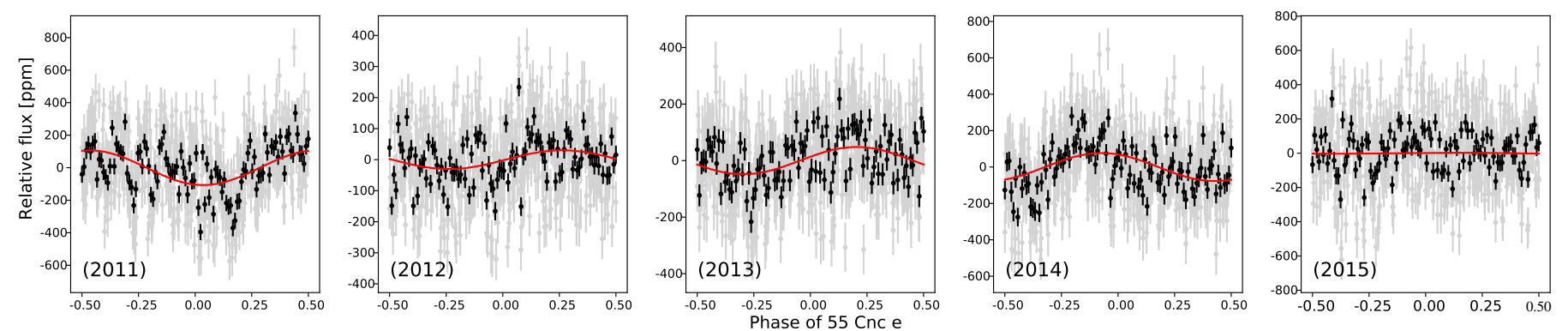

Fig. 5. Light curves detrended by the time-shift procedure, without transits, phase folded at the planet orbital period and binned into 2 (gray) and 10-min (black) intervals.
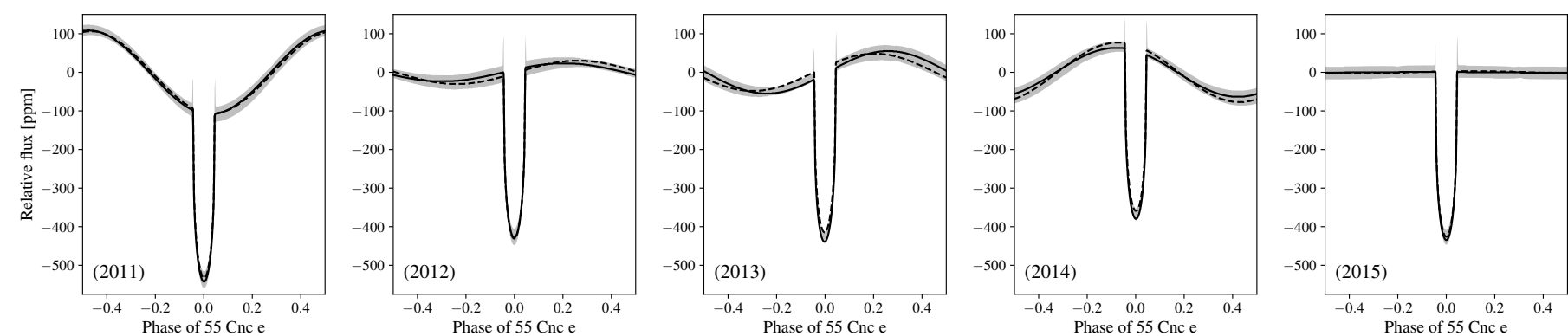

Fig. 6. Best-fitting models resulting from the MCMC analysis as described in Sect. 4, using the light curves detrended by the classical (solid) and time-shift (dashed) procedures. For the latter only, we display the $1 \sigma$ uncertainties (gray shaded area). These uncertainties have been evaluated using $10^{4}$ transit models generated using transit and phase modulation parameters randomly taken from the MCMC posteriors.

where the vectors $\boldsymbol{\alpha}_{\text {mod }}$ and $\boldsymbol{\phi}_{\text {mod }}$ collect all the information about the amplitudes and orbital phases (relative to mid-transit) of the various datasets (simplified hereafter as $\left\{\alpha_{i}, \phi_{i}\right\}$ with $i \in[2011-$ 2015]). In this analysis, we simultaneously estimated 14 free parameters ( 4 for the transit and $5 \times 2$ for the phase variation). Finally, we removed the best-fitting models of transit and phase modulation from the time series and fit the secondary eclipse depth.

Following the reassessed transit parameters of Bourrier et al. (2018a), we assumed an eccentricity of zero for the orbit of the planet and added a prior on the impact parameter $(b=0.39 \pm$ 0.03 ) to help convergence. The results are described below and the best-fitting models for transit and phase modulation for each year are compared in Fig. 6.

\subsection{Updated 55 Cnc e parameters}

With 143 transit events, we precisely estimated the planetary orbital period of $55 \mathrm{Cnc}$ e. This value, found during the first MCMC analysis, is given in the first row of Table 3. Results found on the time series detrended by the classical and timeshift methods are in complete $1 \sigma$ agreement with each other as well as with the period extracted through velocity measurements (Bourrier et al. 2018a). When estimating the flux modulation parameters, the orbital period has to be fixed to ensure the convergence of the second MCMC. The inferred transit and phase modulation parameters obtained during this second fit are given in Tables 3 and 4, respectively. The associated marginal posteriors are reported in Fig. 13 and the best-fitting models are shown in Fig. 6. Once more, we find similar parameters from light curves detrended via the classical and via the time-shift methods. Moreover, the parameters derived from the first and second MCMC agree to better than $1 \sigma$. The most significant difference is for the transit depth that slightly decreases $(<5 \mathrm{ppm})$ between the two runs because of the addition of the phase modulation in the second fit. All values for the transit parameters are in $1 \sigma$ agreement with the values published by Bourrier et al. (2018a) and the previous results based on MOST data by W11 and D14. However, we note that we find slightly shallower transits (at approximately $1 \sigma$ ) than W11 and D14.

\subsection{Phase variations}

Contrary to previous analyses of MOST data (W11, D14), our phase curve model (see Eq. (1)) allows the flux maximum to be offset in time from the planetary occultation. Furthermore, we fit independent phase curve parameters for each year, thus probing temporal variability. Figure 7 illustrates the evolution of the phase modulation from year to year. The phase variation, initially observed in the 2011 dataset by W11, is seen during most of the subsequent years, but its phase and amplitude change. In 2011, the phase variation amplitude is the highest. In 2012, it seems to be attenuated in comparison to the 2011 dataset. In 2013 and 2014, it is present, although with a different phase and amplitude. We find no modulation in 2015, however this dataset is the noisiest and the phase variation might be masked by residual correlated noise (see rms values given in Table 2). Compared to W11, the derived amplitude of the phase modulation measured in the 2011 dataset is smaller ${ }^{1}$ but agrees within $1 \sigma$. When analyzing the light curves of 2011 and 2012 together, and fitting for a common phase modulation for both years, we find an amplitude of $\alpha_{2011+2012}=24_{-7.8}^{+8.7} \mathrm{ppm}$ that agrees with the results ${ }^{2}$ of D14. However, as the modulation changes significantly with time (see Fig. 7), we argue that combining light curves from several years tends to attenuate the observed modulation.

We note that we attempted to probe shorter timescales of the phase curve variability by studying subsets of our five MOST datasets, but the quality of the data at hand are not sufficient to draw meaningful conclusions; i.e., the signal at the planet period

\footnotetext{
1 Winn et al. (2011) found $\alpha_{2011}=168 \pm 70 \mathrm{ppm}$.

2 Dragomir et al. (2014) found $\alpha_{2011+2012}=34_{-11}^{+12} \mathrm{ppm}$.
} 
Table 3. Inferred transit parameters for $55 \mathrm{Cnc}$ e obtained using the light curves detrended by the classical (CM) and time-shift (TM) procedures and their $1 \sigma$ uncertainties.

\begin{tabular}{|c|c|c|c|c|c|c|}
\hline & Parameter & Symbol & Units & Value (CM) & Value (TM) & Bourrier et al. (2018a) \\
\hline \multirow{6}{*}{ 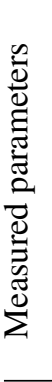 } & Orbital period & $P$ & days & $0.73654530_{-9.5 \times 10^{-7}}^{+6.5 \times 10^{-7}}$ & $0.73654504_{-9 \times 10^{-7}}^{+7.6 \times 10^{-7}}$ & $0.73654737_{-1.44 \times 10^{-6}}^{+1.3 \times 10^{-6}}$ \\
\hline & Transit epoch & $T_{0}-2451545$ & BJD & $4417.0720_{-3.6 \times 10^{-4}}^{+6.1 \times 10^{-4}}$ & $4417.0719_{-5.0 \times 10^{-4}}^{+6.6 \times 10^{-4}}$ & $4417.0712_{-1.4 \times 10^{-3}}^{+1.4 \times 10^{-3}}$ \\
\hline & Planet-to-star radius ratio & $R_{\mathrm{p}} / R_{\mathrm{s}}$ & & $0.01874_{-2.9 \times 10^{-4}}^{+3.9 \times 10^{-4}}$ & $0.01860_{-4.0 \times 10^{-4}}^{+3.2 \times 10^{-4}}$ & $0.0182_{-2 \times 10^{-4}}^{+2 \times 10^{-4}}$ \\
\hline & Impact parameter & $b$ & & $0.3917_{-0.033}^{+0.029}$ & $0.3954_{-0.035}^{+0.027}$ & $0.39_{-0.03}^{+0.03}$ \\
\hline & Transit duration & $t_{\mathrm{d}}$ & days & $0.0648_{-0.9 \times 10^{-3}}^{+1.1 \times 10^{-3}}$ & $0.0654_{-1.4 \times 10^{-3}}^{+0.9 \times 10^{-3}}$ & $0.0634_{-3.7 \times 10^{-4}}^{+3.7 \times 10^{-4}}$ \\
\hline & Eclipse depth & $\delta_{\text {ecl }}$ & ppm & $-9.99_{-10.6}^{+7.5}$ & $-12.88_{-7.6}^{+8.9}$ & \\
\hline \multirow{5}{*}{ 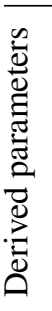 } & Transit depth & $\left(R_{\mathrm{p}} / R_{\mathrm{s}}\right)^{2}$ & ppm & $351.33_{-14.68}^{+11.16}$ & $346.11_{-14.90}^{+11.99}$ & $331.24_{-7.28}^{+7.28}$ \\
\hline & Planetary radius & $R_{\mathrm{p}}$ & $R_{\oplus}$ & $1.8865_{-0.035}^{+0.045}$ & $1.90_{-0.047}^{+0.037}$ & $1.875_{-0.029}^{+0.029}$ \\
\hline & Scaled semimajor axis & $a / R_{\mathrm{S}}$ & & $3.4746_{-7.2 \times 10^{-2}}^{+6.9 \times 10^{-2}}$ & $3.5046_{-9.4 \times 10^{-2}}^{+6.4 \times 10^{-2}}$ & $3.52_{-1.0 \times 10^{-2}}^{+1.0 \times 10^{-2}}$ \\
\hline & Semimajor axis & $a$ & AU & $0.01521_{-3.4 \times 10^{-4}}^{+3.7 \times 10^{-4}}$ & $0.01528_{-3.5 \times 10^{-4}}^{+4.1 \times 10^{-4}}$ & $0.01544_{-5.0 \times 10^{-5}}^{+5.0 \times 10^{-5}}$ \\
\hline & Orbital inclination & $i$ & $\operatorname{deg}$ & $83.56_{-0.62}^{+0.61}$ & $83.72_{-0.74}^{+0.49}$ & $83.59_{-0.44}^{+0.47}$ \\
\hline
\end{tabular}

Notes. The last column indicates the most recent values published in Bourrier et al. (2018a). All the $1 \sigma$ uncertainties were derived using the distribution of the parameter posteriors. The impact parameter was completely determined by the input prior.

Table 4. Inferred parameters of the modulation in flux observed at the planet orbital period.

\begin{tabular}{ccc}
\hline \hline \multicolumn{3}{c}{ Classic } \\
\hline Year & Amplitude $\left(\alpha_{\text {mod }}\right)$ & Phase $\left(\phi_{\text {mod }}\right)$ \\
\hline 2011 & $108.54_{-16.6}^{+12.8} \mathrm{ppm}$ & $0.19_{-0.19}^{+0.27} \mathrm{rad}$ \\
2012 & $23.49_{-9.7}^{+8.2} \mathrm{ppm}$ & $2.20_{-0.47}^{+0.33} \mathrm{rad}$ \\
2013 & $54.96_{-11.2}^{+15.6} \mathrm{ppm}$ & $1.85_{-0.19}^{+0.27} \mathrm{rad}$ \\
2014 & $63.43_{-14.9}^{+14.1} \mathrm{ppm}$ & $3.98_{-0.26}^{+0.21} \mathrm{rad}$ \\
2015 & $-0.77_{-14.7}^{+14.3} \mathrm{ppm}$ & $0.70_{-0.62}^{+0.65} \mathrm{rad}$ \\
\hline
\end{tabular}
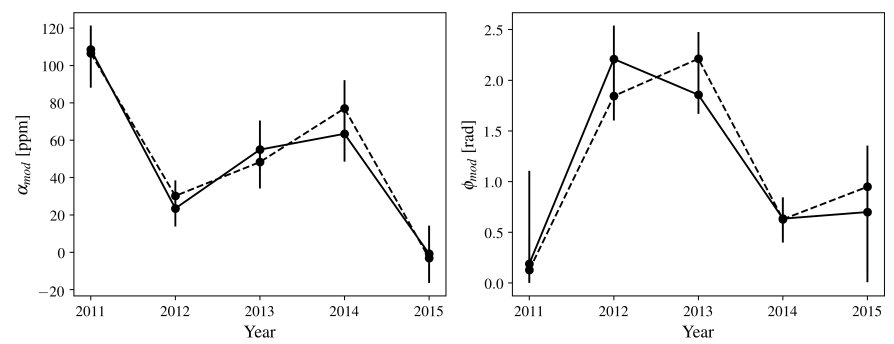

Fig. 7. Evolution of the amplitudes (left) and orbital phases (right) parameters of the modulation in flux observed at the planet orbital period (see Eq. (1)). The solid and dashed lines indicate the light curves detrended by the classical and time-shift procedures, respectively.

only significantly appears when a sufficiently large number of planet periods are phase folded.

\subsection{Secondary eclipse}

To search for the secondary eclipse of $55 \mathrm{Cnc}$ e, we first divided the data by our best-fitting transit and flux modulation models. Then, we performed a final MCMC analysis of the light curve

\begin{tabular}{ccc}
\hline \hline \multicolumn{3}{c}{ Time-shift } \\
\hline Year & Amplitude $\left(\alpha_{\text {mod }}\right)$ & Phase $\left(\phi_{\text {mod }}\right)$ \\
\hline 2011 & $106.52_{-18.4}^{+12.5} \mathrm{ppm}$ & $0.13_{-0.13}^{+0.26} \mathrm{rad}$ \\
2012 & $30.19_{-9.5}^{+8.3} \mathrm{ppm}$ & $1.84_{-0.24}^{+0.36} \mathrm{rad}$ \\
2013 & $48.3_{-14.2}^{+12.1} \mathrm{ppm}$ & $2.21_{-0.29}^{+0.26} \mathrm{rad}$ \\
2014 & $77.01_{-15.3}^{+15.1} \mathrm{ppm}$ & $3.94_{-0.18}^{+0.20} \mathrm{rad}$ \\
2015 & $-3.05_{-13.4}^{+17.3} \mathrm{ppm}$ & $0.94_{-0.30}^{+0.28} \mathrm{rad}$ \\
\hline
\end{tabular}

residuals of all observations. Previous analyses from D14 did not detect the secondary eclipse, and these authors estimated the depth of this eclipse to be $-1_{-22}^{+18} \mathrm{ppm}$ (using the MOST data of 2011 and 2012). Expecting an extremely shallow signature of the planetary eclipse and to ensure obtaining a representative posterior distribution, we allowed the depth parameter to take negative values in our MCMC analysis (no physical meaning).

We find an eclipse depth of $\delta_{\text {ecl }}=-12.88_{-7.6}^{+8.9} \mathrm{ppm}$. Consequently, we do not detect any optical signature of the secondary eclipse of $55 \mathrm{Cnc}$ e, but we can place a $2 \sigma$ limit of $16 \mathrm{ppm}$ on its depth using the posterior distribution. Figure 3 shows the multiyear light curve phase folded on the period of $55 \mathrm{Cnc}$ e, where the phase range of the secondary eclipse is indicated by dotted lines.

We estimated the upper limit on the geometric albedo $\left(A_{\mathrm{g}}\right)$ of 55 Cnc e using the following equation (Rowe et al. 2008):

$\delta_{\text {ecl }}=A_{\mathrm{g}}\left(\frac{R_{\mathrm{p}}}{a}\right)^{2}$.

Given the very close orbit of $55 \mathrm{Cnc}$ e, we compared its predicted thermal contribution with our upper limit on the 

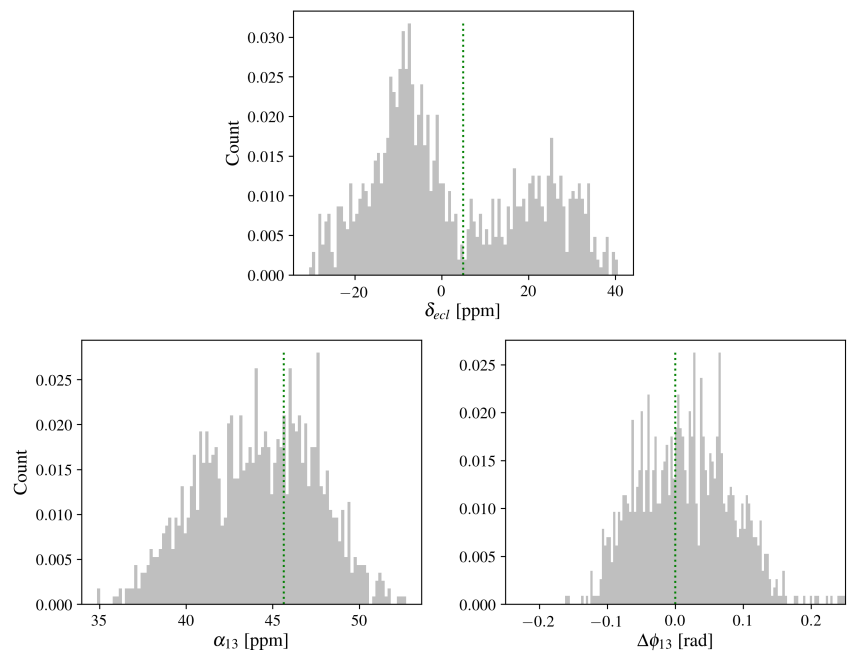

Fig. 8. Normalized distribution of the best-fitting values obtained from injection tests performed for the planetary eclipse (top) and for the phase modulation (bottom). The synthetic eclipse depth has been set to $5 \mathrm{ppm}$ and the phase modulation amplitude to $\alpha_{13}=48.3 \mathrm{ppm}$. Bottom right panel: the difference between the inputs and estimates of the phase parameter $\left(\Delta \Phi_{13}\right)$ as the input phase value is randomly changing from one test to another.

eclipse depth using our $R_{\mathrm{p}} / R_{\mathrm{S}}$ value, a stellar effective temperature of $5172 \pm 18 \mathrm{~K}$ (Yee et al. 2017), and two values for the planet temperature: $2300 \mathrm{~K}$, which is the highest value of the predicted equilibrium temperature (with zero albedo and zero heat redistribution; Crossfield 2012); and $2700 \mathrm{~K}$, which is the hemisphere-averaged value measured by Demory et al. (2016a). The thermal contribution is 0.9 and $4 \mathrm{ppm}$, for each of those two cases, respectively. In this section we derived $2 \sigma$ albedo limits for both cases, but we used and referred to the value corresponding to the measured temperature $(2700 \mathrm{~K})$ throughout the remaining sections of this paper.

We first subtracted the thermal contribution from the $2 \sigma$ limit on $\delta_{\text {ecl }}$, and used the resulting value in Eq. (2). We then derived a $2 \sigma$ lower limit on $R_{\mathrm{p}} / a$ from the corresponding lower limit on $R_{\mathrm{p}} / R_{\mathrm{s}}$ from the second-to-last column of Table 3 , and from the corresponding upper limit on $a / R_{\mathrm{S}}$ from Bourrier et al. (2018a, as their value is in agreement with and more precise than ours). We thus obtained $2 \sigma$ upper limits on $A_{\mathrm{g}}$ of 0.59 and 0.47 assuming planet temperatures of 2300 and $2700 \mathrm{~K}$, respectively.

\subsection{Uncertainties of the inferred parameters}

To validate the uncertainties on both the phase modulation parameters and the eclipse depth, we performed injection tests as done in W11. These tests consist in injecting a synthetic signal (either the secondary eclipse or the phase modulation) in the light curve residuals and performing an MCMC analysis of these synthetic light curves to estimate the parameters of interest. For both cases, we used the 2013 data only to save on computation time and performed 1000 individual injections. We used an eclipse depth of $\delta_{\mathrm{ecl}}=5 \mathrm{ppm}$ and a phase modulation amplitude of $\alpha_{13}=45.63 \mathrm{ppm}$. When injecting secondary eclipses, the time at mid-eclipse is randomly chosen between $\left[t_{0}-t_{\mathrm{d}} / 2, t_{0}+P-t_{\mathrm{d}} / 2\right]$. When injecting a phase modulation, the phase parameter $\Phi_{13}$ is randomly chosen between $[-2 \pi, 2 \pi]$.

Figure 8 shows the distribution of the best-fitting values for the recovered eclipse depth (top) and the parameters $\alpha_{13}$ and $\Phi_{13}$ (bottom). For the secondary eclipse depth, we find a bimodal distribution around the true injected value (green dashed line). This distribution is centered around the true value, but is clearly not Gaussian. This illustrates the influence of the correlated noise still present in the reduced light curves. Depending on the injected secondary eclipse timing, its depth is over - or under estimated as a consequence of the average level of correlated noise at this orbital phase. From these tests, we estimate a realistic uncertainty on the occultation depth of up to $35 \mathrm{ppm}$ (measured as the maximum dispersion around the true value of 5 ppm).

For the phase modulation, the distribution of the retrieved values for amplitude and phase are centered around the true values. The widths of the two distributions are $7 \mathrm{ppm}$ and $0.2 \mathrm{rad}$ for amplitude and phase, respectively. These results show that the impact of the remaining correlated noise is less significant on timescales longer than the satellite orbital period; i.e., they do not affect the modulation in flux related to the planet orbit, for which we also have many more photons.

Beside the injection tests, we also tested whether reliable uncertainties can be obtained by scaling the input errors. The MCMC code used in this work uses the $\chi^{2}$ as its merit function, however this implicitly assumes white Gaussian noise. As correlated noise persists in the data even after the corrections described in Sect. 3.1, this would lead to largely underestimated errors on the derived parameters. Therefore, we scale our photometric errors. We use the $\beta_{r}$ factor that compares the ratio of standard deviations evaluated on the binned and raw residuals (Winn et al. 2008; Gillon et al. 2010). We tested bin intervals between 5 and 20 minutes and scaled the error bars by the highest value found. We find that a relatively large factor of $\beta_{\mathrm{r}}=2.262$ allows us to obtain similar uncertainties on the phase curve parameters as those derived using injection tests.

The uncertainties given in Tables 3 and 4 were derived using this $\beta_{\mathrm{r}}$ method. We note that for the secondary eclipse search, we used the errors scaled during the second MCMC analysis.

\section{Search for transits of $55 \mathrm{Cnc} \mathrm{b}, \mathrm{c}, \mathrm{f}$, and d}

There are four other known planets in the $55 \mathrm{Cnc}$ system that have been detected via radial velocity measurements (Fischer et al. 2008; Dawson \& Fabrycky 2010). Given that the innermost one, i.e., planet e, transits the host star, there is a non-negligible probability that one or more of the others may transit as well. Their masses and orbital periods span wide ranges (Dawson \& Fabrycky 2010), forming an extrasolar multiple planet system that is moderately similar to our solar system. $55 \mathrm{Cnc} \mathrm{f}$ holds particular interest because it spends about $74 \%$ of its eccentric orbit within the habitable zone of the system (von Braun et al. 2011); we note that this statement may no longer be true as the recent re-evaluation of the eccentricity is now consistent with zero (Bourrier et al. 2018a).

Using parameters given in Table 3 of Bourrier et al. (2018a), we computed the predicted transit times during our MOST observations for the five innermost planets. Figure 9 shows the MOST data phase folded at the orbital period of the planets with the 1,2 , and $3 \sigma$ uncertainties of their time at mid-transit. When using the MCMC routines described above to search for transits of each of these planets, we come up empty. However, assuming the innermost planets $b$ and $c$ were transiting, we can give an upper limits on their planetary radii. Considering $b=0.5$ as the nominal value (because the orbital inclination of each of these planets' orbit is unknown), we predict an upper limit of $2.15 R_{\oplus}$ for planet $55 \mathrm{Cnc} \mathrm{b}$ and $2.56 R_{\oplus}$ for planet $55 \mathrm{Cnc} \mathrm{c}$ (assuming a limiting $\mathrm{S} / \mathrm{N}$ of 40 as planet e). 

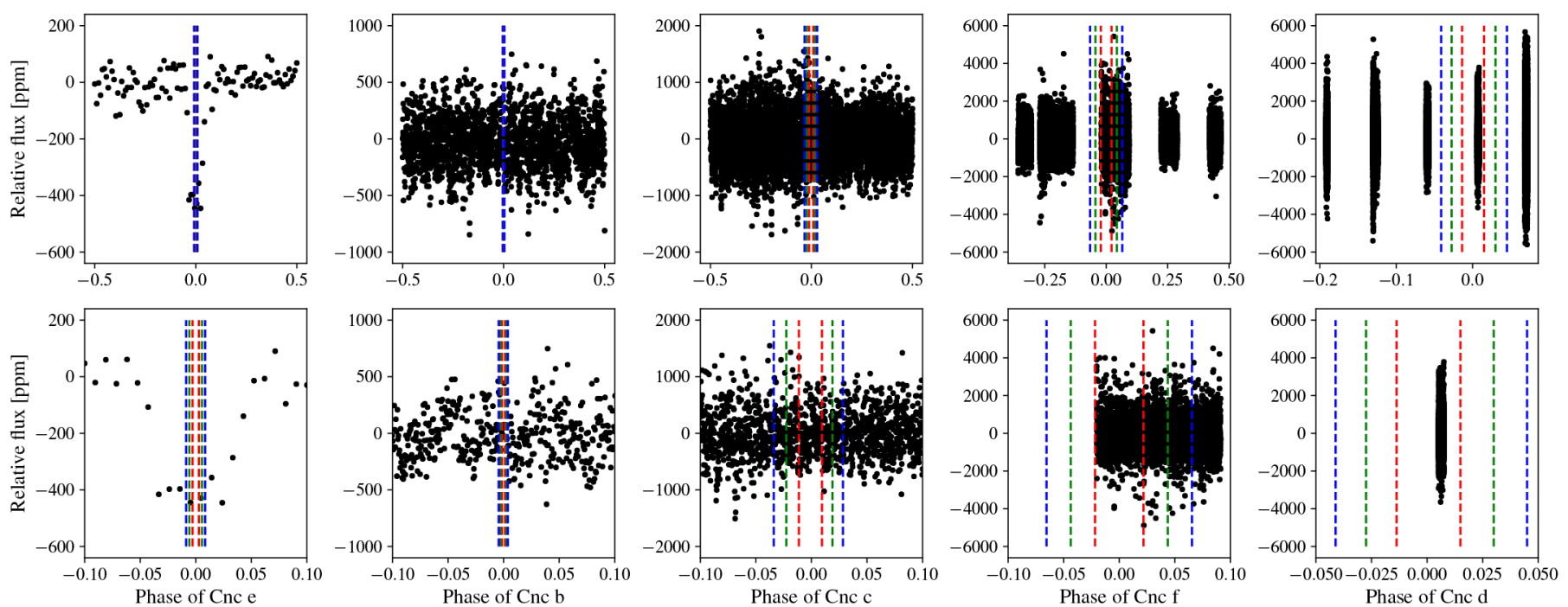

Fig. 9. From left to right: light curves binned into 5-min intervals, phase folded at the orbital period of planet e, b, c, f, and d. The vertical lines indicate the 1,2, and $3 \sigma$ uncertainties of the time of mid-transit (red, green, and blue, respectively). Bottom panels: zoomed-in views of the mid-time transit event for each planet.

\section{Discussion}

\subsection{Albedo of 55 Cnce}

While we do not detect a secondary eclipse of $55 \mathrm{Cnc}$ e, we can consider the 0.47 upper limit on its albedo in the context of other albedo measurements in the literature. Thanks to Kepler and K2, it has been determined that hot Jupiters are typically dark and likely cloudless (i.e., $A_{\mathrm{g}}<0.2$; Esteves et al. 2015; Angerhausen et al. 2015). A few hot Jupiters have $A_{\mathrm{g}}$ values between 0.2 and 0.35 (Esteves et al. 2015; Angerhausen et al. 2015; Demory et al. $2011 \mathrm{~b}$ ), indicating that a small fraction of these planets have clouds.

However, 55 Cnc e should be compared to planets of its own size. Albedo measurements of the Kepler close-in superEarth and Neptune sample as a whole have revealed that these worlds are somewhat less dark than hot Jupiters, but still have $A_{\mathrm{g}}$ values generally below 0.3 (Demory 2014; Sheets \& Deming 2017; Jansen \& Kipping 2018). One exception is the hot rocky exoplanet Kepler-10b (Batalha et al. 2011). Rouan et al. (2011) suggested $\mathrm{ThO}_{2}$ particles dispersed in $\mathrm{Al}_{2} \mathrm{O}_{3}-\mathrm{CaO}$ lava as a possible origin for the relatively high (0.32) geometric albedo of the planet.

If the surface of $55 \mathrm{Cnc}$ e is molten (Demory et al. 2016a) and assuming that at visible wavelengths we can view all the way to its surface, Kane et al. (2011) predicted a secondary eclipse depth of $20 \mathrm{ppm}$ using an $A_{\mathrm{g}}$ value of 0.6 . However, recent preliminary laboratory measurements of specular reflection from molten lava and quenched glass (a product of rapidly cooled lava) suggest an upper limit on the albedo of such a planetary surface of 0.1 (Zahra Essack, MIT, priv. comm.). While we note that the albedo of molten rock depends on the composition of the mantle of the planet, which is unknown for $55 \mathrm{Cnc} \mathrm{e}$, a molten surface unobstructed by an atmosphere (at the wavelengths probed by the MOST data) remains possible within our $A_{\mathrm{g}}$ upper limit.

While our constraints on the geometric albedo of $55 \mathrm{Cnc}$ e do not rule out any of the most likely atmospheric composition models (e.g., $\mathrm{CO}, \mathrm{CO}_{2}, \mathrm{H}_{2} \mathrm{O}, \mathrm{N}_{2}, \mathrm{O}_{2}, \mathrm{HCN}$; Angelo \& $\mathrm{Hu} 2017$; Miguel 2019), we can provide a first test of the model proposed by Tamburo et al. (2018) to explain the previously observed IR secondary eclipse variability (Demory et al. 2016b). Tamburo et al. (2018) found that refractory particulates produced by volcanic activity, at times lofted high in the atmosphere, could obscure the surface of the planet and potentially explain the decrease in observed thermal emission. According to this model, $55 \mathrm{Cnc}$ e would have a higher albedo (between 0.4 and 1.0) when its surface is obscured by the refractory particulates. In this scenario, this stage of the variability (e.g., when Spitzer $4.5 \mu \mathrm{m}$ secondary eclipse depth was at its lowest) was observed in 2012. The photometric precision of the MOST 2012 light curve alone is not sufficient to set a meaningful constraint on $A_{\mathrm{g}}$ for that year. Nevertheless, our global constraint of $A_{\mathrm{g}}<0.47$ (averaged over all five MOST light curves) rules out most of the 0.4-1.0 range, and tentatively suggests that the model proposed by Tamburo et al. (2018) may not explain the observed $4.5 \mu \mathrm{m}$ secondary eclipse variability. Ultimately, high-precision observations with the upcoming CHaracterising ExOPlanet Satellite (CHEOPS) space telescope (Broeg et al. 2013) would provide improved constraints on, and possibly an actual determination of, the albedo of $55 \mathrm{Cnc}$ e.

\subsection{Possible origins of the phase modulations}

In this study, we analyzed five sequences of MOST data of 55 Cnc e obtained between 2011 and 2015 and searched for photometric modulation in phase with the period of planet e. We assumed a modulation that was periodic and estimated its parameters (amplitude and phase) for the five individual light curves. We detected a phase modulation and find that it is variable from year to year. Intriguingly, the amplitude of this modulation in flux is too large to be due to scattered light from the planet. We note that our measurements of the phase amplitude are also higher than the predictions by Kane et al. (2011) who assumed various scenarios for the planet; the most optimistic is that of a lava world with a modulation amplitude $<20 \mathrm{ppm}$. Consequently, the observed modulation must have a different origin.

\subsubsection{Instrumental origin}

First, it has been proposed by W11 that the variation may be due to instrumental noise. To verify this, we analyzed the light 

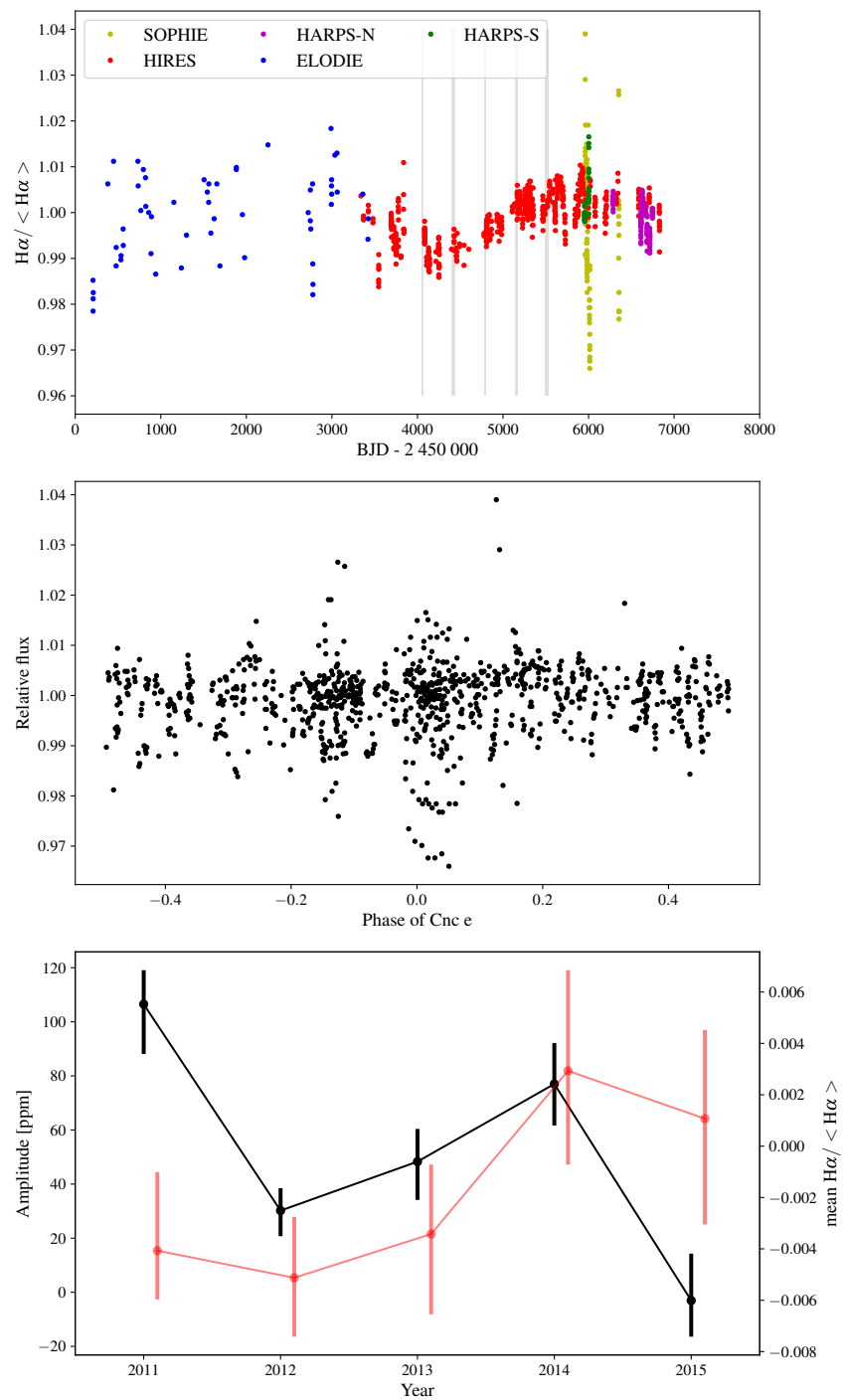

Fig. 10. Top: $\mathrm{H}-\alpha$ activity index of the $55 \mathrm{Cnc}$ A star (see Fig. 2 of Bourrier et al. 2018a, second panel) and MOST observations dates (gray). Middle: combined $\mathrm{H}-\alpha$ data phase folded at the planet orbital period. Bottom: amplitudes of the modulation (see Table 4) measured on MOST observations (black, left $y$-axis) and mean values of the $\mathrm{H}-\alpha$ stellar activity indicator (red, right $y$-axis) evaluated around dates close to the MOST observations. The error bars on the mean $\mathrm{H}-\alpha$ values are taken as the minimum and maximum values of the index in the considered year. We note the $\mathrm{H}-\alpha$ values have been slightly shifted in time for visibility. We observe the increase/decrease of the modulation in phase with the increase/decrease of the stellar activity indicator.

curve of the nearby star $53 \mathrm{Cnc}$, a bright giant star falling on the MOST CCD during the observations of $55 \mathrm{Cnc}$. We applied the same detrending procedures to the light curves of $53 \mathrm{Cnc}$ and do not find any flux modulation at the timescale of the orbital period of $55 \mathrm{Cnc}$ e. While we are pretty confident that the modulation observed in the $55 \mathrm{Cnc}$ is not due to instrumental artifacts, this has to been confirmed by other long-term observations in the optical wavelength range. Assuming this modulation is indeed related to the $55 \mathrm{Cnc}$ system, we can invoke multiple hypothetical physical scenarios to explain its origin.

\subsubsection{Stellar variability}

We considered the possibility of variability of the star as the source of the phase modulation signal. The rotation period of $55 \mathrm{Cnc} \mathrm{A}$ is $\sim 40$ days and the lifetime of star spots on solar-type

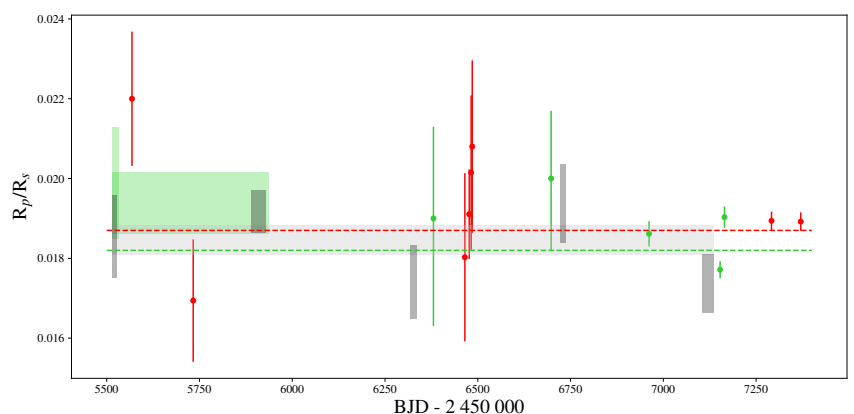

Fig. 11. Reproduction of Fig. 15 of Bourrier et al. (2018a) representing the planet-to-star radius ratio measured over time with various instruments. Observations in the optical are shown in green. Chronologically, we show values from Gillon et al. (2012) and D14 with MOST; from de Mooij et al. (2014) with Alhambra Faint Object Spectrograph and Camera (ALFOSC) and from Bourrier et al. (2018a) with the Space Telescope Imaging Spectrograph (STIS) spectrograph onboard the Hubble Space Telescope (HST). Our measurements derived from MOST and resulting from the time-shift procedure are shown in black (individual points and combined light curves). Observations in IR are represented in red: Demory et al. (2016a) with Spitzer and Tsiaras et al. (2016) with Wide Field Camera 3 (WFC3) onboard HST. Following Bourrier et al. (2018a), the dashed green line shows the value obtained from the fit to the three combined STIS visits and the dashed red line shows the value obtained from the fit to the combined Spitzer visits (Demory et al. 2016b). The values represented by rectangles indicate radius ratios that have been estimated over an extended period of time.

stars ranges from 10 to 350 days (Namekata et al. 2019). These timescales are too long for spot-related stellar variability to be the source of the signal. We also considered stellar pulsations, but in Sun-like stars $p$-mode oscillations have periods of just 5-10 min (Di Mauro 2016), which is much too short to be the source of the observed modulations at the period of $55 \mathrm{Cnc}$ e. We conclude that neither star spots nor stellar pulsations can explain the $55 \mathrm{Cnc}$ e phase modulation signal.

\subsubsection{Star-planet interaction}

As proposed by W11, this signal can be the signature of an interaction between the host star and planet e. For example, from ultraviolet observations Bourrier et al. (2018b) proposed that the interaction of the planet with the stellar magnetic field inside the corona might trigger coronal rain. The material would be accreted onto the star along the magnetic field lines, and cool and emit at optical wavelengths (see discussion in Sect. 4.3 of Bourrier et al. 2018b). In this scenario, the variability in the observed modulation would be due to the variability of the stellar corona with time and to exchanges of variable amounts of material over time. To validate this scenario, we looked for modulations of the $\mathrm{H}-\alpha$ stellar activity indicator obtained over the last $\approx 20 \mathrm{yr}$ (Bourrier et al. 2018a) in phase with the planetary orbital period, but without success (see Fig. 10). This may be because the sampling and time coverage may be too poor to access the presence of such a modulation at the level allowed by the data quality. However, it is also possible that the signal is smeared out by the variation in the phase offsets we observe in the modulation from year to year, which is on timescales much shorter than the whole data coverage, even possibly shorter than the stellar rotation period. For this reason, we considered the subseries of stellar activity indicator measurements that are temporally close to the MOST observations. Figure 10 shows that there is indeed a similarity in the temporal behavior between the activity index and the amplitude of the flux modulation. 

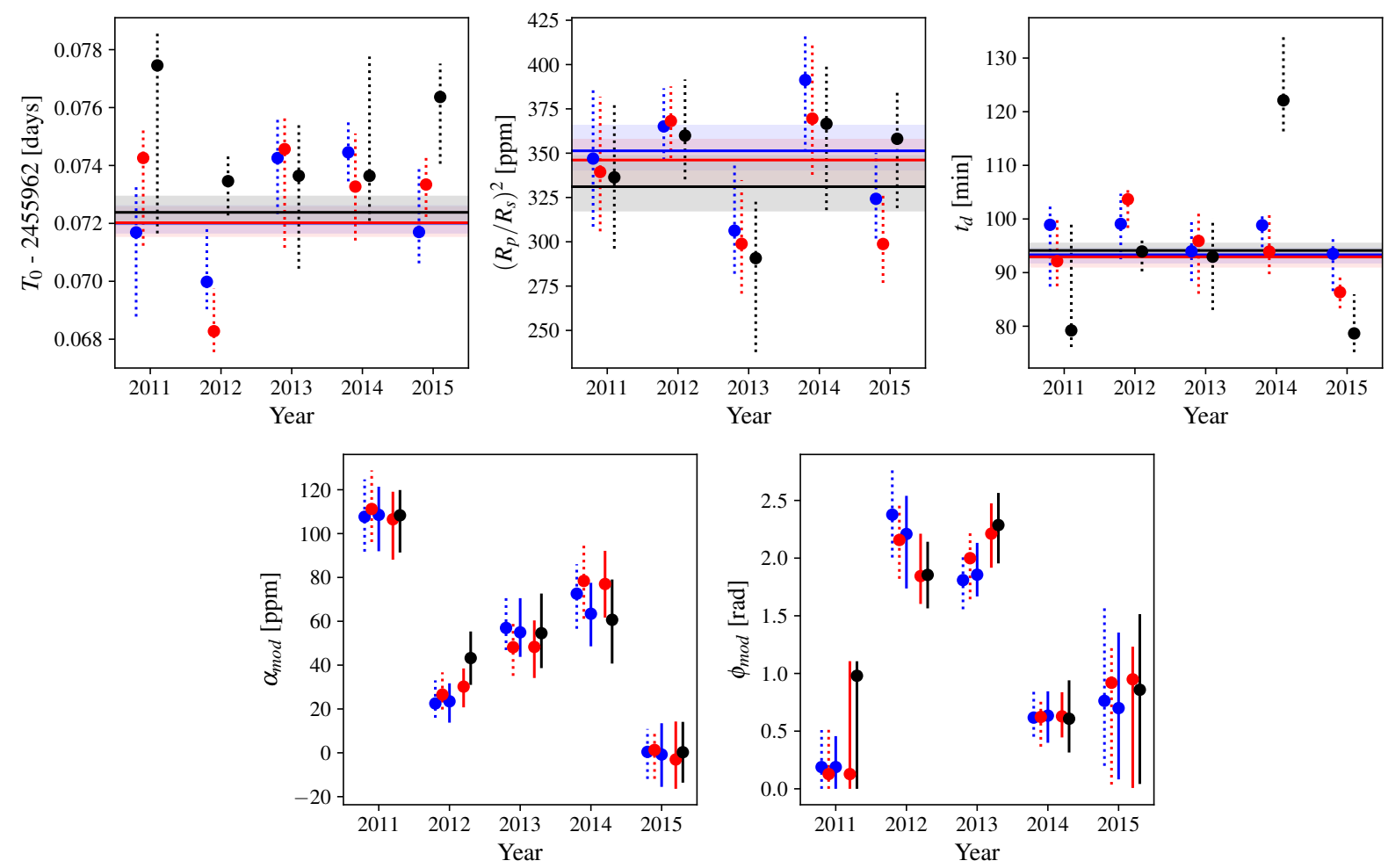

Fig. 12. From left to right: estimated parameters of the 55 Cnc e transit $\left(T_{0},\left(R_{\mathrm{p}} / R_{\mathrm{s}}\right)^{2}, t_{\mathrm{d}}\right)$ and of the modulation in flux $\left(\alpha_{\bmod }\right.$ and $\left.\phi_{\bmod }\right)$. In all panels, dotted points indicate the best-fitting values derived from each of the 5 MOST data analyzed separately using the classical and time-shift detrending procedures and values obtained using the light curve not corrected for the stray-light systematics (i.e., resulting only from the pre-whitening step; see Sect. 3.1). In the three first panels, the horizontal solid lines represent the values derived from the combined light curves (see Table 3 and Sect. 4.1) with their $1 \sigma$ uncertainties (shaded area). In the two last panels, the solid points represent the modulation parameters derived during the analysis of these combined light curves (see Table 4 and Sect. 4.2).

A supporting argument for the presence of star-planet interactions comes from the results of Folsom et al. (2019), who employed spectropolarimetry to derive the strength and geometry of the stellar magnetic field. Their 3D stellar wind modeling based on the surface magnetic field map indicated that the orbit of the planet lies entirely inside the Alfvén surface of the stellar wind. This implies that the star and the planet are magnetically connected and that the planet could influence the stellar wind such that the interaction can reach the stellar surface. If this scenario is correct, the observed flux modulation could be evidence of plasma exchange through reconnecting magnetic field lines of the star and planet.

The interaction could then lead to the formation of a spot on the stellar surface that rotates in phase with the planetary orbital motion, hence unrelated to the stellar rotation period (Shkolnik et al. 2003; Strugarek et al. 2015). Such a scenario has already been proposed to explain the optical flux modulation observed for the $\tau$ Boo (Walker et al. 2008) and CoRoT-2 (Pagano et al. 2009) systems, as well as in X-rays for HD 17156 (Maggio et al. 2015). If a spot is present, calculations done for hot Jupiters have shown that the activity should be phased with the planetary orbital period and present a phase offset (Lanza 2012) similar to what was recently detected by Cauley et al. (2018). Assuming this argument also holds for systems hosting lower mass planets and the quality and sampling of the data is high enough, the interaction should be detectable by analyzing the temporal behavior of magnetic activity indicators (Strugarek et al. 2019). Our results shown in Fig. 10 suggest that the interaction may indeed be detectable with simultaneous photometric and spectroscopic high-quality observations, the former covering the whole planet orbital period and the latter covering multiple activity indicators.

\subsubsection{Transiting circumstellar dust torus}

Alternatively, the phase modulation in flux could be the signature of a transiting circumstellar dust torus. This dust cloud could also exchange material with the planet itself, which might originate from volcanism at the surface of 55 Cnc e (analogous to Io's cold plasma torus; Krüger et al. 2003). The density and optical depth of the torus changes over time. Thus, both the transit depth and flux modulation vary (see Fig. 11). This scenario would be in line with the variability observed in the IR secondary eclipse depth (Demory et al. 2016b; Tamburo et al. 2018). Moreover, if the material of this torus indeed originates from the planet, it could be alimented from material (typically dust) inside the planetary Roche lobe $\left(\sim 4.94-5.22 R_{\oplus}\right)$, which itself is highly irradiated and subject to intense tidal forces. In this paradigm, the proportion of scattered light also depends on the spatial distribution of the dust inside the Roche lobe and the position of the planet along its orbit. If the distribution of scattering material material evolved, the phase curve would as well.

\subsection{Search for temporal variations in the transit parameters of 55 Cnce}

By independently analyzing the five light curves taken from 2011 to 2015 , we observe some variations of the transit parameters from year to year. This is shown in Fig. 12 for the transit and modulation parameters. We observe a variability in the transit depth that seems in agreement with Demory et al. (2016b) and Bourrier et al. (2018a), who suggest variations over timescales of days or weeks. This is shown in Fig. 11 (reproduction of Fig. 15 of Bourrier et al. 2018a with our measurements added). 


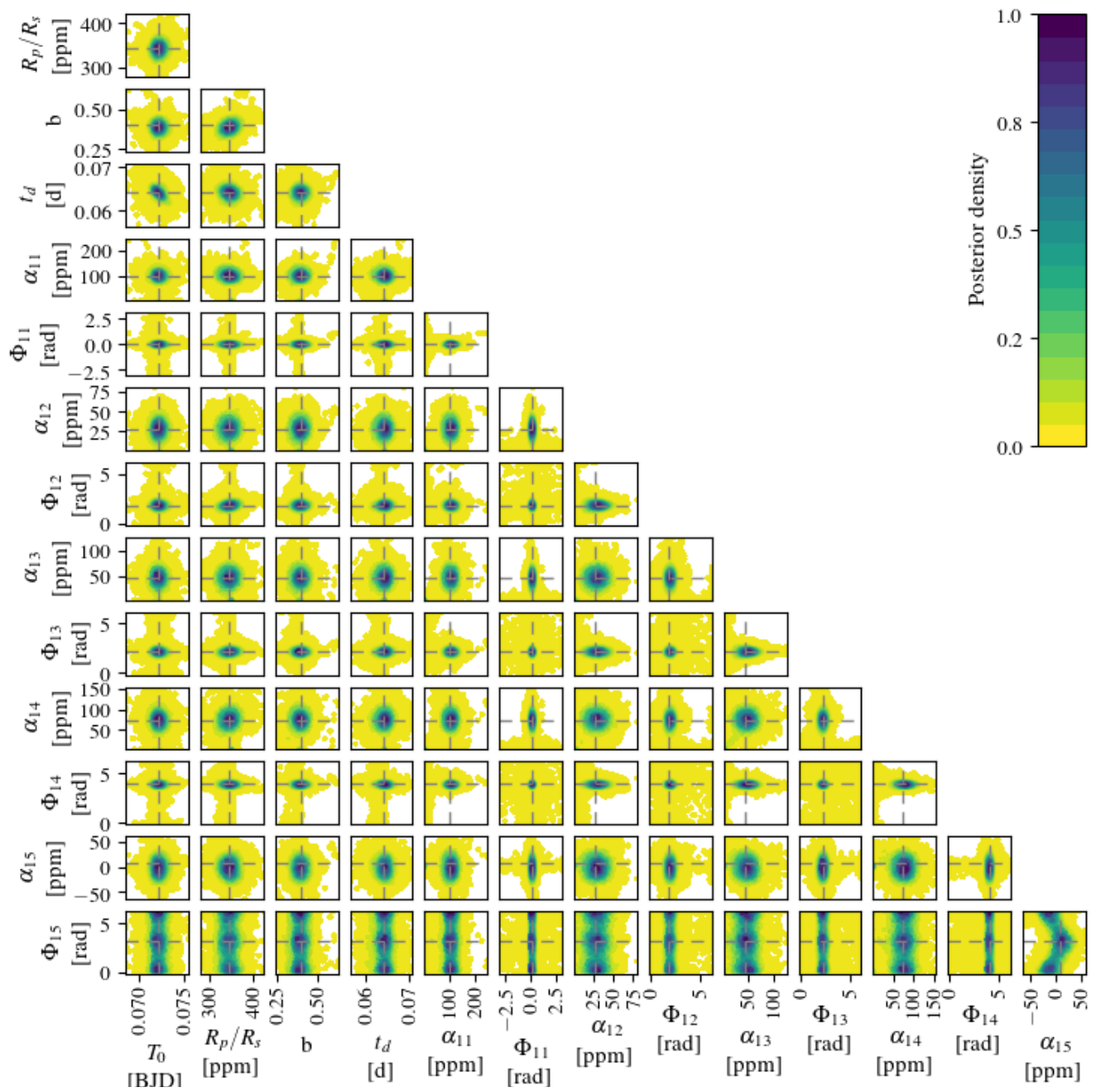

Fig. 13. Pairwise marginal posteriors of the MCMC analysis for the $55 \mathrm{Cnc}$ e planet and the phase variation modeled as described in Eq. (1). For visibility, we plot $T 0-4417$ and fold the $\Phi_{11}$ parameter over $\pm \pi$. We found a double maxima for the $\Phi_{15}$ parameter around 0 [mod $\left.2 \pi\right]$ and $\pi$, which is meaningless as the corresponding modulation amplitude $\alpha_{15}$ is roughly null.

However, while we measure a maximum deviation of the transit depth up to $40 \mathrm{ppm}$, all the measurements are within the $1 \sigma$ values of each other (see Fig. 12). Hence, the transit depth variability is not beyond the expected statistical fluctuations of our MOST observations.

In Fig. 12, we also compare values obtained before and after the stray-light correction procedures (see Sect. 3.2). As expected, we observe that the stray-light correction improves the precision and even the accuracy of the transit parameters (see top panels), and by extension, the constraint on the eclipse depth. However, the impact on the inferred parameters of the modulation is less pronounced (see bottom panels).

\section{Conclusions}

We analyzed five sequences of MOST observations spanning several weeks of observations taken between 2011 and 2015. We carried out a careful reduction of the raw light curves following current MOST reduction techniques. In addition, we developed a new method, based on the cross-correlation of shorter subsequences to improve the correction of the stray-light noise.

We performed MCMC analyses of the combined detrended MOST light curves and derived the transit, secondary-eclipse, and phase-modulation parameters. We find transit parameters that are consistent with the most recent values given in
Bourrier et al. (2018a). The secondary eclipse remains undetected in the MOST observations, but it allows us to constrain the albedo of $55 \mathrm{Cnc}$ e to an upper limit of 0.47 . We confirm the detection of the optical modulation in flux discovered by Winn et al. (2011) at the planet period and detect it at four additional epochs. Intriguingly, we find that its amplitude and phase are variable. At this point, we can only speculate about the origin of the effect. Simultaneous observations at various wavelengths could help to distinguish the origin of this variability that may be linked to the observed modulation observed in far-ultraviolet (Bourrier et al. 2018b) and IR wavelengths (Demory et al. 2016b; Tamburo et al. 2018). We argue that additional observations in the optical with Transiting Exoplanet Survey Satellite (TESS; Ricker et al. 2014) and CHEOPS will be extremely valuable for our understanding of this mysterious planet. Finally, we do not detect any transit for planets b, c, f, and $d$ in the MOST observations.

Acknowledgements. S.S., M.L., P.C., and L.F. acknowledge support from the Austrian Research Promotion Agency (FFG) under project 859724 "GRAPPA". D.D. acknowledges support provided by NASA through Hubble Fellowship grant HSTHF2-51372.001-A awarded by the Space Telescope Science Institute, which is operated by the Association of Universities for Research in Astronomy, Inc., for NASA, under contract NAS5-26555. V.B. acknowledges support by the Swiss National Science Foundation (SNSF) in the frame of the National Centre for Competence in Research PlanetS, and has received funding from the European 
Research Council (ERC) under the European Union's Horizon 2020 research and innovation programme (project Four Aces; grant agreement No 724427) The authors thank Apurva Oza for a careful reading of an earlier version of the manuscript and the anonymous referee for his/her very useful comments.

\section{References}

Adams, E. R., Seager, S., \& Elkins-Tanton, L. 2008, ApJ, 673, 1160 Angelo, I., \& Hu, R. 2017, AJ, 154, 232

Angerhausen, D., DeLarme, E., \& Morse, J. A. 2015, PASP, 127, 1113

Batalha, N. M., Borucki, W. J., Bryson, S. T., et al. 2011, ApJ, 729, 27

Batygin, K., \& Laughlin, G. 2015, Proc. Natl. Acad. Sci., 112, 4214

Bourrier, V., Dumusque, X., Dorn, C., et al. 2018a, A\&A, 619, A

Bourrier, V., Ehrenreich, D., Lecavelier des Etangs, A., et al. 2018b, A\&A, 615, A117

Broeg, C., Fortier, A., Ehrenreich, D., et al. 2013, EPJ Web Conf., 47, 03005

Cauley, P. W., Shkolnik, E. L., Llama, J., Bourrier, V., \& Moutou, C. 2018, AJ, 156,262

Chiang, E., \& Laughlin, G. 2013, MNRAS, 431, 3444

Crossfield, I. J. M. 2012, A\&A, 545, A97

Cubillos, P., Harrington, J., Loredo, T. J., et al. 2017, AJ, 153, 3

Davis, T. A., \& Wheatley, P. J. 2009, MNRAS, 396, 1012

Dawson, R. I., \& Fabrycky, D. C. 2010, ApJ, 722, 937

de Mooij, E. J. W., López-Morales, M., Karjalainen, R., Hrudkova, M., \& Jayawardhana, R. 2014, ApJ, 797, L21

Demory, B.-O. 2014, ApJ, 789, L20

Demory, B.-O., Gillon, M., Deming, D., et al. 2011a, A\&A, 533, A114

Demory, B.-O., Seager, S., Madhusudhan, N., et al. 2011b, ApJ, 735, L12

Demory, B.-O., Gillon, M., de Wit, J., et al. 2016a, Nature, 532, 207

Demory, B.-O., Gillon, M., Madhusudhan, N., \& Queloz, D. 2016b, MNRAS, 455, 2018

Di Mauro, M. P. 2016, in Frontier Research in Astrophysics II, 29

Dorn, C., Harrison, J. H. D., Bonsor, A., \& Hands, T. O. 2018, MNRAS, 484 712

Dragomir, D., Matthews, J. M., Eastman, J. D., et al. 2013, ApJ, 772, L2

Dragomir, D., Matthews, J. M., Winn, J. N., \& Rowe, J. F. 2014, IAU Symp., 293, 52

Ehrenreich, D., \& Désert, J.-M. 2011, A\&A, 529, A136

Ehrenreich, D., Bourrier, V., Bonfils, X., et al. 2012, A\&A, 547, A18

Esteves, L. J., De Mooij, E. J. W., \& Jayawardhana, R. 2015, ApJ, 804, 150

Fischer, D. A., Marcy, G. W., Butler, R. P., et al. 2008, ApJ, 675, 790

Folsom, C. P., Fionnagáin, D. O., Fossati, L., et al. 2019, MNRAS, submitted

Fressin, F., Torres, G., Charbonneau, D., et al. 2013, ApJ, 766, 81

Fulton, B. J., \& Petigura, E. A. 2018, AJ, 156, 264

Fulton, B. J., Petigura, E. A., Howard, A. W., et al. 2017, AJ, 154, 109

Gandolfi, D., Barragán, O., Livingston, J. H., et al. 2018, A\&A, 619, L10

Gillon, M., Lanotte, A. A., Barman, T., et al. 2010, A\&A, 511, A3

Gillon, M., Demory, B.-O., Benneke, B., et al. 2012, A\&A, 539, A28

Hansen, B. M. S., \& Murray, N. 2012, ApJ, 751, 158

Jansen, T., \& Kipping, D. 2018, MNRAS, 478, 3025

Jin, S., \& Mordasini, C. 2018, ApJ, 853, 163

Jones, H. R. A., Paul Butler, R., Tinney, C. G., et al. 2002, MNRAS, 333, 871
Kane, S. R., Gelino, D. M., Ciardi, D. R., Dragomir, D., \& von Braun, K. 2011, ApJ, 740, 61

Kane, S. R., Wittenmyer, R. A., Hinkel, N. R., et al. 2016, ApJ, 821, 65

Kite, E. S., Fegley, Jr. B., Schaefer, L., \& Gaidos, E. 2016, ApJ, 828, 80

Krüger, H., Geissler, P., Horányi, M., et al. 2003, Geophys. Res. Lett., 30, 2101

Kubyshkina, D., Fossati, L., Erkaev, N. V., et al. 2018, A\&A, 619, A151

Lanza, A. F. 2012, A\&A, 544, A23

Lecavelier des Etangs, A. 2007, A\&A, 461, 1185

Lendl, M., Cubillos, P. E., Hagelberg, J., et al. 2017, A\&A, 606, A18

Maggio, A., Pillitteri, I., Scandariato, G., et al. 2015, ApJ, 811, L2

Mandel, K., \& Agol, E. 2002, ApJ, 580, L171

Matthews, J. M. 2004, BAAS, 36, 1563

Mayor, M., Marmier, M., Lovis, C., et al. 2011, ArXiv e-prints [arXiv:1109.2497]

Miguel, Y. 2019, MNRAS, 482, 2893

Mordasini, C., Alibert, Y., Georgy, C., et al. 2012, A\&A, 547, A112

Motalebi, F., Udry, S., Gillon, M., et al. 2015, A\&A, 584, A72

Namekata, K., Maehara, H., Notsu, Y., et al. 2019, ApJ, 871, 187

Owen, J. E., \& Wu, Y. 2017, ApJ, 847, 29

Pagano, I., Lanza, A. F., Leto, G., et al. 2009, Earth Moon Planets, 105, 373

Petigura, E. A., Howard, A. W., \& Marcy, G. W. 2013, Proc. Natl. Acad. Sci., 110, 19273

Pont, F., Zucker, S., \& Queloz, D. 2006, MNRAS, 373, 231

Poppenhaeger, K., \& Schmitt, J. H. M. M. 2011, ApJ, 735, 59

Ricker, G. R., Winn, J. N., Vanderspek, R., et al. 2014, Proc. SPIE Conf. Ser., 9143, 914320

Ridden-Harper, A. R., Snellen, I. A. G., Keller, C. U., et al. 2016, A\&A, 593, A129

Rogers, L. A., \& Seager, S. 2010, ApJ, 712, 974

Rogers, L. A., Bodenheimer, P., Lissauer, J. J., \& Seager, S. 2011, ApJ, 738, 59

Rouan, D., Deeg, H. J., Demangeon, O., et al. 2011, ApJ, 741, L30

Rowe, J. F., Matthews, J. M., Seager, S., et al. 2006, ApJ, 646, 1241

Rowe, J. F., Matthews, J. M., Seager, S., et al. 2008, ApJ, 689, 1345

Rucinski, S. M., Walker, G. A. H., Matthews, J. M., et al. 2004, PASP, 116, 1093

Savitzky, A., \& Golay, M. J. E. 1964, Anal. Chem., 36, 1627

Sheets, H. A., \& Deming, D. 2017, AJ, 154, 160

Shkolnik, E. L., \& Llama, J. 2018, Signatures of Star-Planet Interactions (Berlin: Springer), 20

Shkolnik, E., Walker, G. A. H., \& Bohlender, D. A. 2003, ApJ, 597, 1092

Strugarek, A., Brun, A. S., Matt, S. P., \& Réville, V. 2015, ApJ, 815, 111

Strugarek, A., Brun, A. S., Donati, J. F., Moutou, C., \& Réville, V. 2019, ApJ, 881,136

Tamburo, P., Mandell, A., Deming, D., \& Garhart, E. 2018, AJ, 155, 221

Tsiaras, A., Rocchetto, M., Waldmann, I. P., et al. 2016, AJ, 820, 99

Van Eylen, V., Agentoft, C., Lundkvist, M. S., et al. 2018, MNRAS, 479, 4786

Vogt, S. S., Burt, J., Meschiari, S., et al. 2015, ApJ, 814, 12

von Braun, K., Boyajian, T. S., ten Brummelaar, T. A., et al. 2011, ApJ, 740, 49

Walker, G., Matthews, J., Kuschnig, R., et al. 2003, PASP, 115, 1023

Walker, G. A. H., Croll, B., Matthews, J. M., et al. 2008, A\&A, 482, 691

Winn, J. N., Holman, M. J., Torres, G., et al. 2008, ApJ, 683, 1076

Winn, J. N., Matthews, J. M., Dawson, R. I., et al. 2011, ApJ, 737, L18

Wright, J. T., \& Miller, B. 2015, IAU Gen. Assem., 29, 2258453

Yee, S. W., Petigura, E. A., \& von Braun, K. 2017, ApJ, 836, 77 


\section{Appendix A: Long-term variability affecting the 55 Cancri light curve: signature of the stellar activity}

During the pre-whitening stage (see Sect. 3.1), we observe a long-term variation in each of the light curves. This variation is shown in Fig. A.1. We observe variations over timescales close to half of the stellar rotation period (38.8/2 day) but that changes in both amplitude and phase from year to year (see right column). When binning the observations into two-hour intervals (see left column), we measure a dispersion around the mean value of $656,1471,1116,774$, and $1733 \mathrm{ppm}$ for the 2011-2015 datasets, respectively. We attribute these variations to the evolution of the stellar activity as they are not strictly increasing as would be expected in case of instrumental systematics. We note that the stray-light systematics discussed in Sect. 3.2 are clearly visible in the three first datasets. Correcting for the long-term variation before correcting the stray-light systematics, as done in this study contrary to previous studies of W11 and D14, avoids the introduction of an offset between the one-day sequences used in the analysis described in Sect. 3.2. 

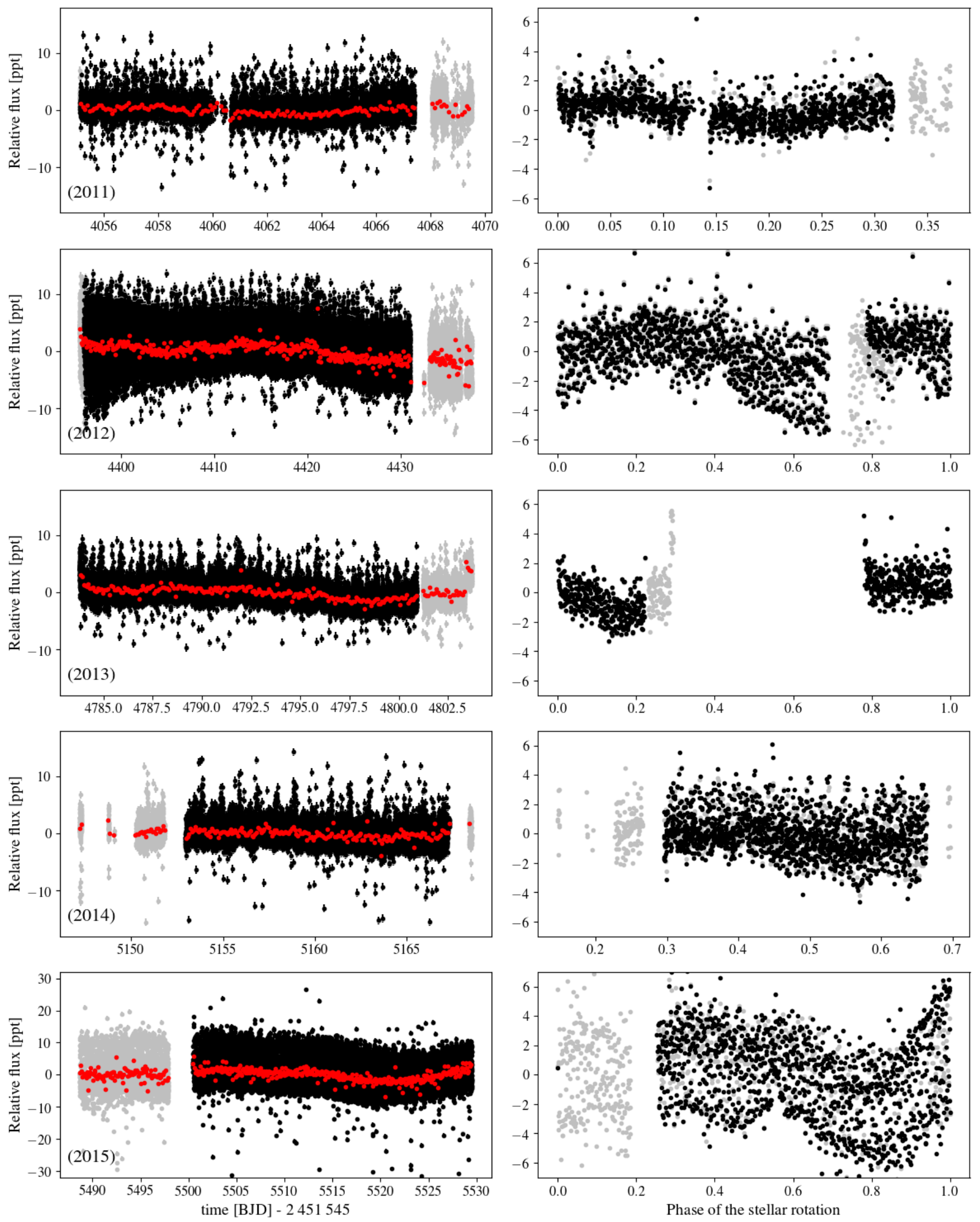

Fig. A.1. From top to bottom: light curves taken in 2011-2015 obtained before the long-term variation correction in the pre-whitening stage (see Sect. 3.1). The $y$-axis is in part-per-thousand. The removed sections of the light curves are shown in gray. Left column panels: represent the light curve as a function of time. The long-term variation is well observed on the data binned at two-hour intervals (red). Right column panels: represent these flux phase folded at the stellar rotation period of 38.8 days (binned into 30-min intervals). We note the different scale on the $y$-axis of the 2015 plot on the bottom. 